\title{
Safflor Yellow B Attenuates Ischemic Brain Injury via Downregulation of Long Noncoding AK046177 and Inhibition of MicroRNA-134 Expression in Rats
}

\author{
Chaoyun Wang $\mathbb{D}^{1}{ }^{1}$ Hongzhi Wan, ${ }^{2}$ Qiaoyun Wang, ${ }^{2}$ Hongliu Sun, ${ }^{2}$ Yeying Sun, ${ }^{2}$ \\ Kexin Wang, ${ }^{3}$ and Chunxiang Zhang $\mathbb{1}^{4}$ \\ ${ }^{1}$ Hearing and Speech Institute, Binzhou Medical University, Yantai 264003, China \\ ${ }^{2}$ School of Pharmaceutical Sciences, Binzhou Medical University, Yantai 264003, China \\ ${ }^{3}$ Third Class of Senior High School, No. 2 Middle School of Yantai Shandong, Yantai, China \\ ${ }^{4}$ Department of Biomedical Engineering, School of Medicine, University of Alabama at Birmingham, Birmingham, AL, USA
}

Correspondence should be addressed to Chaoyun Wang; ytwcy@163.com and Chunxiang Zhang; zhangchx999@163.com

Received 8 July 2019; Revised 11 March 2020; Accepted 29 April 2020; Published 4 June 2020

Guest Editor: Reggiani Vilela Gonçalves

Copyright ( 92020 Chaoyun Wang et al. This is an open access article distributed under the Creative Commons Attribution License, which permits unrestricted use, distribution, and reproduction in any medium, provided the original work is properly cited.

\begin{abstract}
Stroke breaks the oxidative balance in the body and causes extra reactive oxygen species (ROS) generation, leading to oxidative stress damage. Long noncoding RNAs (lncRNAs) and microRNAs play pivotal roles in oxidative stress-mediated brain injury. Safflor yellow B (SYB) was able to effectively reduce ischemia-mediated brain damage by increasing antioxidant capacity and inhibiting cell apoptosis. In this study, we investigated the putative involvement of lncRNA AK046177 and microRNA-134 (miR-134) regulation in SYB against ischemia/reperfusion- (I/R-) induced neuronal injury. I/R and oxygen-glucose deprivation/reoxygenation (OGD/R) were established in vivo and in vitro. Cerebral infarct volume, neuronal apoptosis, and protein expression were detected. The effects of SYB on cell activity, cell respiration, nuclear factor erythroid 2-related factor 2 (Nrf2), antioxidant enzymes, and ROS were evaluated. I/R or OGD/R upregulated the expression of AK046177 and miR-134 and subsequently inhibited the activation and expression of CREB, which caused ROS generation and brain/cell injury. SYB attenuated the effects of AK046177, inhibited miR-134 expression, and promoted CREB activation, which in turn promoted Nrf2 expression, and then increased antioxidant capacities, improved cell respiration, and reduced apoptosis. We suggested that the antioxidant effects of SYB were driven by an AK046177/miR-134/CREB-dependent mechanism that inhibited this pathway, and that SYB has potential use in reducing or possibly preventing I/R-induced neuronal injury.
\end{abstract}

\section{Introduction}

Stroke is an important cerebrovascular disease that afflicts many people worldwide and frequently causes death or long-term disability [1]. Ischemic stroke is the most common type, accounting for about $80 \%$ of all strokes $[2,3]$. Brain injury is caused by disruption of blood flow to the brain and is characterized by oxidative stress. In addition, reoxygenation resulting from the restoration of blood flow exacerbates tissue damage [4].

Pathophysiologically, ischemia and reperfusion can inhibit the activity of endogenous antioxidant enzymes and promote the overproduction of reactive oxygen species (ROS) [5-7]. Previous studies have shown that antioxidants significantly reduce ischemic damage through the inhibition of ROS production [8-11]. Nuclear factor erythroid 2related factor 2 (Nrf2) is a reduction-oxidation- (redox-) sensitive transcription factor that binds to antioxidant response elements (ARE) and activates the transcription of antioxidant enzymes. Studies have shown that cysteine residues on protein Keap1 are oxidized by ROS, leading to the release and activation of Nrf2 [12,13]. Thus, Nrf2 is a useful therapeutic target for reducing or preventing ROS damage in the brain following ischemia/reperfusion (I/R) injury. Cyclic 
AMP (cAMP) response element-binding protein (CREB) is a leucine zipper transcription factor that inhibits ROS generation and suppresses severe ischemic injury by upregulating brain-derived neurotrophic factor (BDNF) and $\mathrm{Bcl}-2$ [14, 15].

MicroRNAs (miRNAs) are endogenous, short $(\approx 22$ nucleotides), noncoding single-strand RNAs that regulate gene expression at the posttranscriptional level by influencing the translation of specific target mRNAs. Recent research revealed that a variety of miRNAs play important roles in ischemic injury through the modulation of cellular redox reactions and mitochondrial function [16]. For example, downregulation of miR-134 enhances $\mathrm{Bcl}-2$ expression and alleviates ischemic injury by regulating CREB activity [17].

Long noncoding RNAs (lncRNAs) play key roles in various cellular contexts under both physiological and pathological conditions, and they are involved in diverse biological processes such as RNA processing, modulation of apoptosis and invasion, and chromatin modification [18-20]. AK046177 is a 606-base pair (bp) noncoding RNA sequence derived from a gene sequence (from 116850844 to 116851448) located on chromosome 13.

Safflower yellow is the flavonoid compound extracted from Carthamus tinctorius L., which includes the components hydroxysafflor yellow A (HSYA) and safflor yellow B (SYB). It has been shown to effectively reduce oxidative stress-mediated damage $[21,22]$. A study by Wang et al. demonstrated that HSYA significantly increases antioxidant enzyme activity by activating the cAMP/PKA signaling pathway [23]. SYB (Figure 1(a)) is a yellow amorphous powder with a purity of more than $98 \%$ by HPLC, and it is water soluble and has demonstrated protective effects in neuronal injury models induced by oxidative stress [24, 25]. However, its effect on brain injury induced by I/R remains to be investigated. This study tested whether SYB reduces I/R-mediated brain injury, and evaluated its potential mechanisms by studying changes in the expression of AK046177, miR-134, Nrf2, and CREB.

\section{Materials and Methods}

2.1. Experimental Design and Cerebral I/R. Male SpragueDawley rats (11 months of age), weighing about 350 to $400 \mathrm{~g}$, were purchased from the Experimental Animal Department of Shandong Luye Pharmaceutical Co. Ltd. (Yantai, China). All animals (5 rats per cage) were bred in a temperature-controlled animal facility with a $12 \mathrm{~h}$ light/dark cycle.

Animals were randomly assigned to the following 6 groups: sham $(n=19), \mathrm{I} / \mathrm{R}(n=19), \mathrm{I} / \mathrm{R}+\mathrm{AK} 046177$ siRNA oligo $(n=19), \mathrm{I} / \mathrm{R}+\mathrm{SYB}(n=19), \mathrm{I} / \mathrm{R}+\mathrm{SYB}+\mathrm{miR}-134$ agomir $(n=19)$, and I/R+AK046177 siRNA oligo+miR-134 agomir $(n=19)$.

Rats were anesthetized with $10 \%$ chloral hydrate in $0.9 \%$ $\mathrm{NaCl}\left(300 \mathrm{mg} / \mathrm{kg}\right.$, i.p.) and placed on a $37^{\circ} \mathrm{C}$ temperaturecontrolled heating pad. AK046177 siRNAs and miR-134 agomir were synthesized by GenePharma (Shanghai, China). Except for the sham group, all rats from the other groups were treated as follows: every morning at 9 o'clock, $2 \mathrm{ml}$ of
SYB or saline was administered intravenously at a dose of $6 \mathrm{mg} / \mathrm{kg}$ continuously for 3 days prior to I/R injury, and $4 \mu \mathrm{l}$ of AK046177 siRNA oligo (100 nM) or miR-134 agomir $(80 \mathrm{nM})$ with Lipofectamine was administered via intracerebroventricular infusion half an hour before ischemia

Middle cerebral artery occlusion (MCAO) was performed according to the monofilament method as described by Longa et al. and Macrae [26, 27]. After $1 \mathrm{~h}$ of ischemia followed by $23 \mathrm{~h}$ of reperfusion, a single experimenter blinded to treatment condition determined the neurological deficit score of each rat according to Longa et al.'s previously validated five-point scale, described below [26]. Following neurological evaluation, rats were decapitated under deep anesthesia and brains were removed. Some whole brains were sectioned and infarct volume was measured by staining with 2,3,5-triphenyl-tetrazolium chloride (TTC; 1.5\%). The right part from the same region of the cerebral cortex (the damaged hemisphere for $\mathrm{MCAO}$ ) and other brain tissues were isolated and stored at $-80^{\circ} \mathrm{C}$ in a freezer. All animals were treated in accordance with the National Institutes of Health Guide for Care and Use of Laboratory Animals (NIH Publications No. 8023, revised 1996). Animal care and experimental procedures were approved by the Ethics Committee on Animal and Human Experimentation of Binzhou Medical University (approval no. 2016077).

2.2. Neurological Tests. After 23 hours of reperfusion, eight rats of each group were subjected to a modified neurological examination designed to evaluate total motor deficit [28]. Briefly, rats were placed on a $10-20 \mathrm{~cm}$ horizontal screen, which was rotated from a horizontal to vertical position. The length of time each rat remained on the vertical screen was recorded and scored as 1 point per $5 \mathrm{~s}$, to a maximum of $15 \mathrm{~s}$ ( 3 points). Rats were then placed at the center of a horizontal wooden rod, and the length of time they remained on the rod was recorded and scored as 1 point per $10 \mathrm{~s}$, to a maximum of $30 \mathrm{~s}$ ( 3 points). Rats were placed on a horizontal rope, and the length of time each rat remained on the rope was recorded and scored as 1 point per 2 seconds, to a maximum of $6 \mathrm{~s}$ ( 3 points). A total motor score (TMS) was calculated based on the results of these assessments (maximum 9 points).

2.3. TUNEL Staining. After 23 hours of reperfusion, three rats of every group were anesthetized and intracardially perfused with $4 \%$ paraformaldehyde (PFA) in $0.1 \mathrm{M}$ phosphatebuffered saline (PBS; pH 7.4). Following perfusion, the brains were removed and postfixed overnight in $4 \% \mathrm{PFA}$ at $4^{\circ} \mathrm{C}$. The brains were embedded in paraffin and sliced into coronal sections ( $5 \mu \mathrm{m}$; Leica Biosystems, Wetzlar, Germany). Apoptosis was detected using an in situ cell death detection kit (Roche, Germany), in accordance with the manufacturer's protocol. Samples were stained with DAPI for $5 \mathrm{~min}$, following which apoptotic (TUNEL-positive) cells were visualized as localized bright red signals on a black background using a DMR fluorescence microscope (Leica Microsystems, Wetzlar, Germany). An apoptotic index (AI) was determined as the ratio of apoptotic cells to the total number of cells, averaged from 3 sections per animal. 


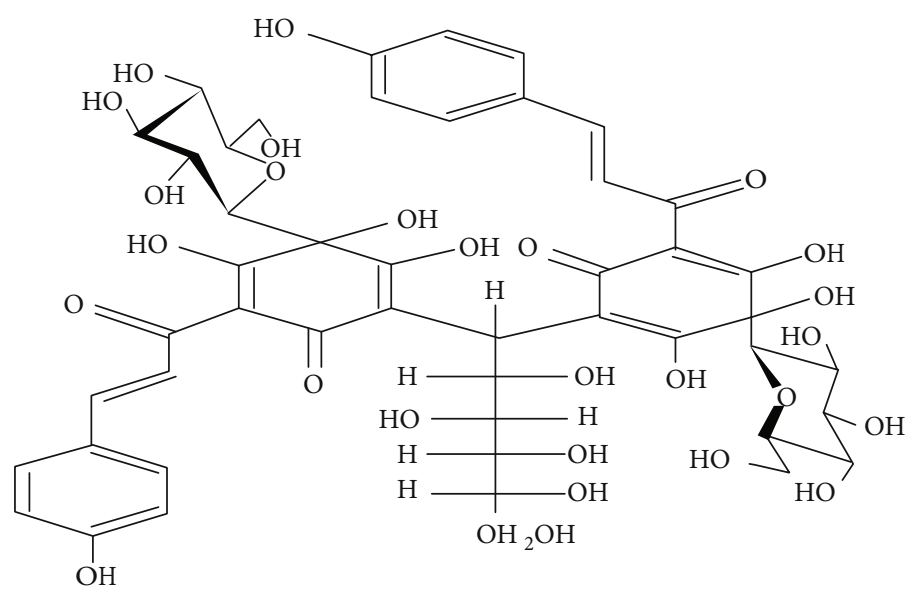

(a)

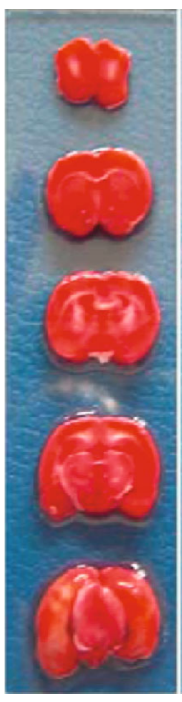

a

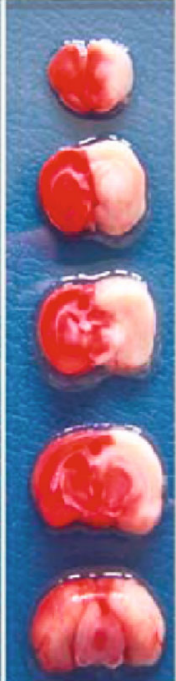

b

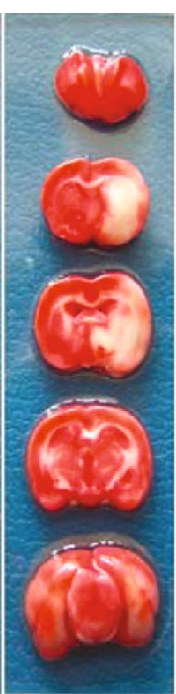

C

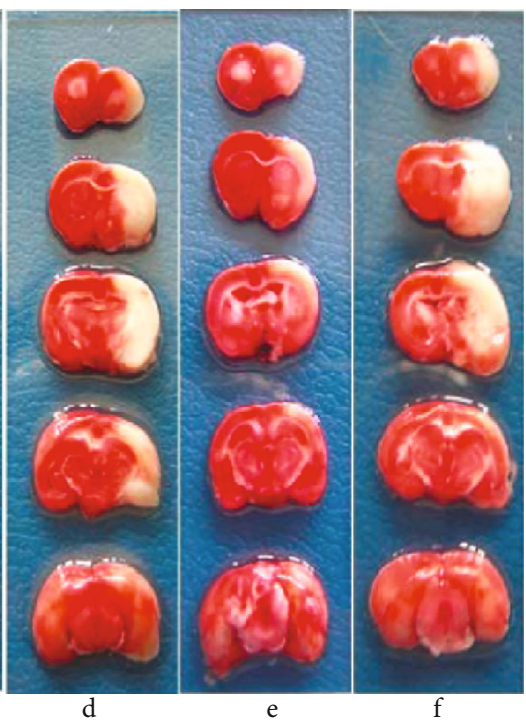

(b)

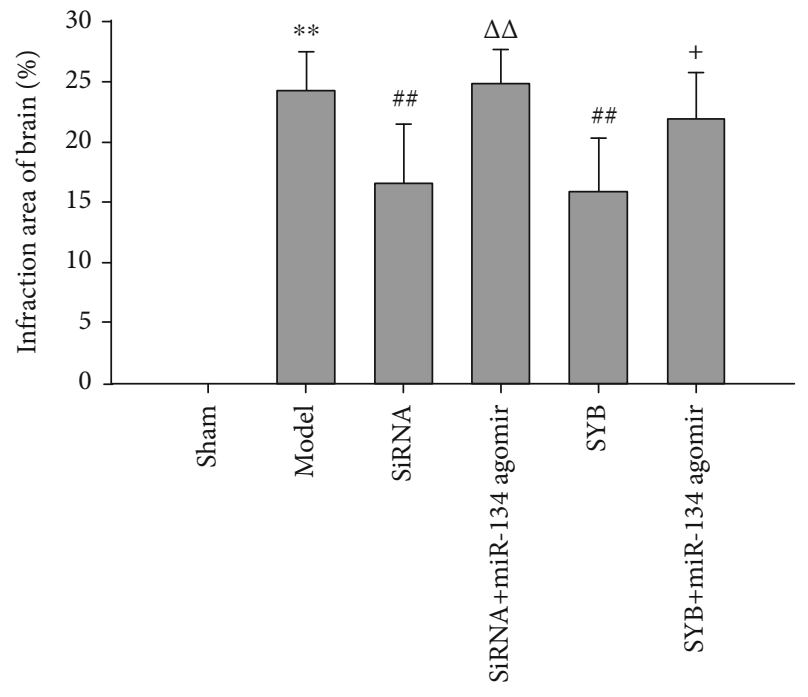

(c)

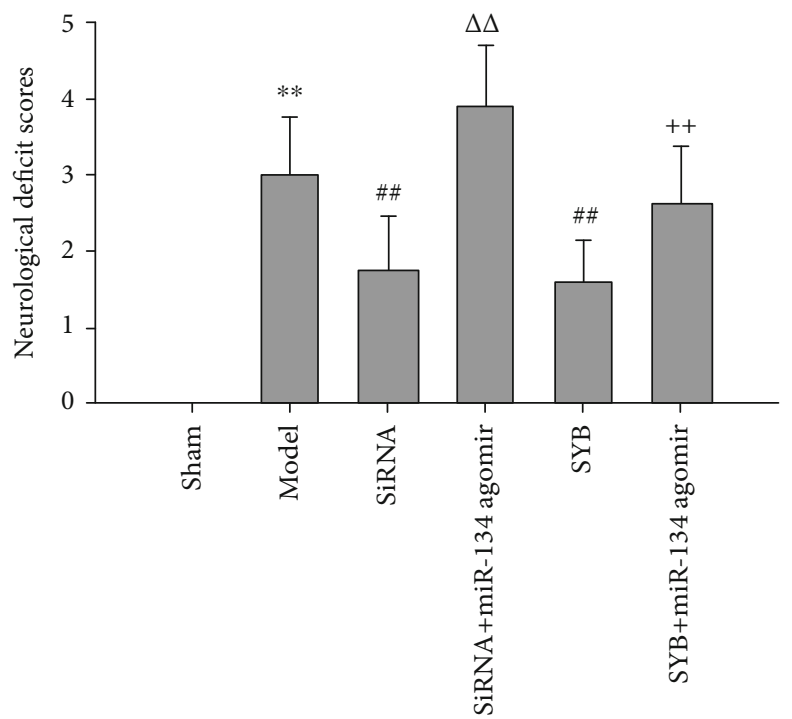

(d)

FIgure 1: Continued. 


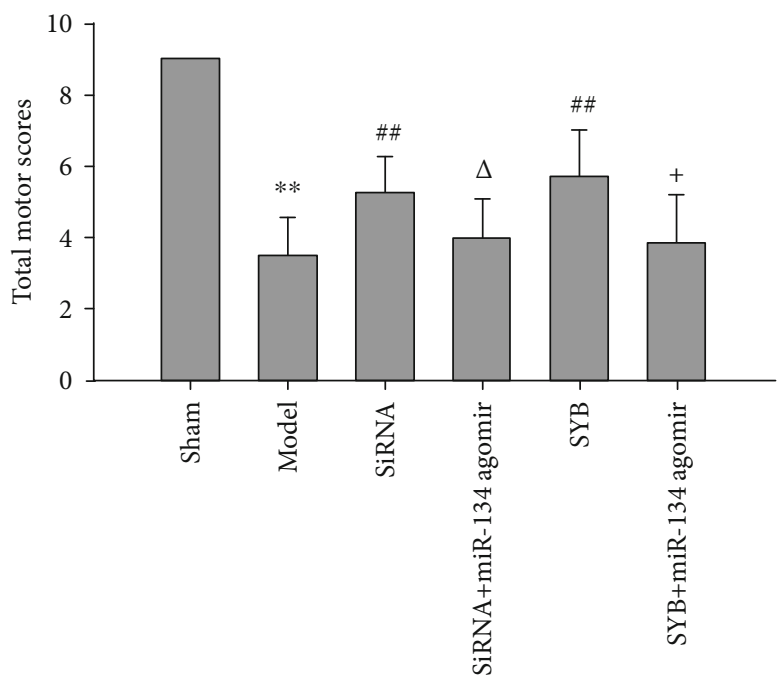

(e)

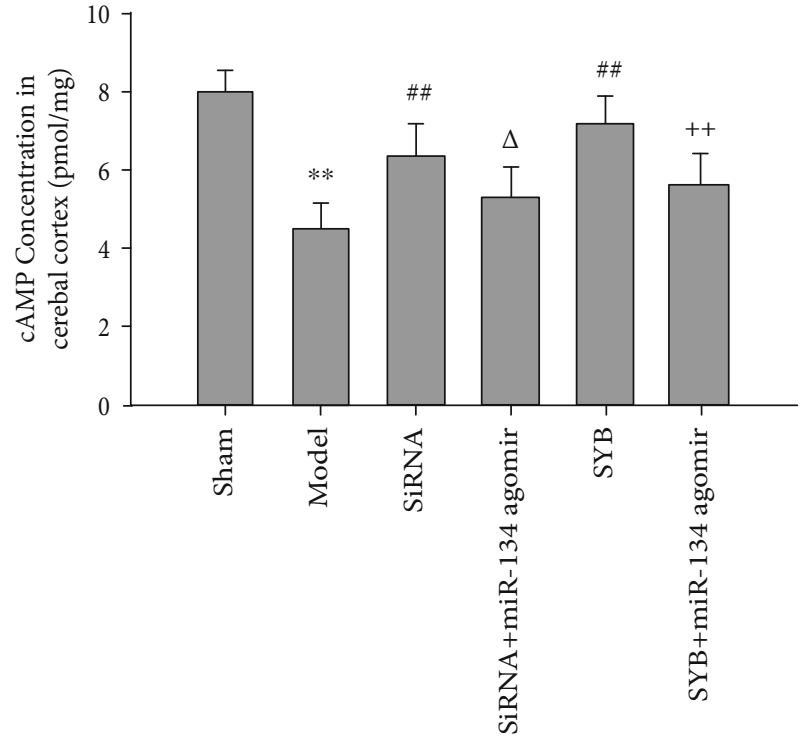

(f)

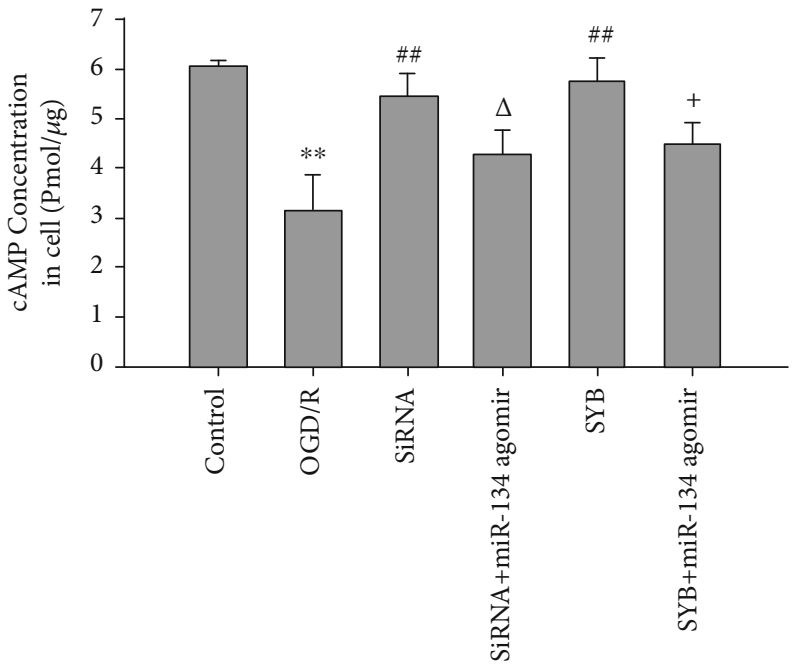

(g)

FIgURE 1: Effect of safflor yellow B on neurological deficit score, infarction area, total motor score, and cAMP level. (a) Chemical structure of safflor yellow B. As shown in (b)-(e), rats were divided into six groups: sham, ischemia/reperfusion (I/R), AK046177 siRNA, AK046177 siRNA+miR-134 agomir, SYB, SYB+miR-134 agomir. Except for the sham group, all rats in the other groups had established ischemia for $1 \mathrm{~h}$ followed by reperfusion for $23 \mathrm{~h}$. SYB and saline were administrated by tail vein continuously for three days before treatment with I/R, and miR-134 agomir and AK046177 siRNA were given via intracerebroventricular injection. The neurological deficit score of each rat was obtained according to Longa's method. Infarct volumes were measured by staining brain sections with 2,3,5-triphenyltetrazolium chloride. (a) represents pathological changes of cerebral infarction ((a) sham; (b) I/R; (c) AK046177 siRNA; (d) AK046177 siRNA+miR-134 agomir; (e) SYB; (f) SYB+miR-134 agomir). (b) represents neurological deficit scores. (c) represents the infarction area. (d) represents total motor scores $(n=8)$. As shown in (f)-(g), cAMP levels in the cerebral cortex and primary fetal cortical cells were detected using a ${ }^{125}$ I-radioimmunoassay according to the method described by the manufacturer. Data are presented as mean \pm S.D. ( $n=8$ in tissues; $n=3$ in cells). One-way ANOVA test was used to determine statistical significance. ${ }^{* *} P<0.01 v s$. the sham group or the control group, ${ }^{\# \#} P<0.01 v$ s. the I/R group or the OGD/R group, ${ }^{\Delta} P<0.05$ or ${ }^{\Delta \Delta} P<0.01 v s$. the AK046177 siRNA group, and ${ }^{+} P<0.05$ or ${ }^{++} P<0.01 v$ s. the SYB group.

2.4. Cell Culture. Primary cortical cells from the cerebral cortex of fetal rats (E16-18) were grown in Dulbecco's Minimal Essential Medium (DMEM) supplemented with 10\% $(v / v)$ fetal bovine serum, $1 \%$ penicillin/streptomycin, and $3.7 \mathrm{~g} / \mathrm{l}$ $\mathrm{NaHCO}_{3}$. Before the initiation of the experiment, the cells were seeded in dishes and precultured for 3 to 5 days at $37^{\circ} \mathrm{C}$ in a humidified incubator, with $5 \% \mathrm{CO}_{2}$ and $95 \%$ air. They were then cultured and divided into 7 groups: control, oxygen-glucose deprivation/reoxygenation (OGD/R), AK046177 siRNA (OGD/R+siRNA), AK046177 siRNA+agomiR-134 (OGD/R+siRNA+agomiR-134), SYB (OGD/R +SYB), SYB+agomiR-134 (OGD/R+SYB+agomiR-134), and negative control (OGD/R+NC). All groups excluding the control group were cultured in glucose-free DMEM under 
hypoxic conditions $\left(1 \% \mathrm{O}_{2} / 94 \% \mathrm{~N}_{2} / 5 \% \mathrm{CO}_{2}\right)$ at $37^{\circ} \mathrm{C}$ for $4 \mathrm{~h}$. Thereafter, media in all groups was replaced with normal DMEM, and culturing continued for $20 \mathrm{~h}$ of reoxygenation under normoxic conditions (95\% air/5\% $\mathrm{CO}_{2}$ ). Cells were pretreated with SYB (final concentration of $0.5 \mathrm{mmol} / \mathrm{l}$ ), AK046177 siRNA (80 nmol/l), and agomiR-134 (50 nmol/l) for $24 \mathrm{~h}$ prior to OGD/R. siRNA, agomiR-134, or control antagomir were transfected into primary cortical cells for 24 h using Lipofectamine RNAiMAX Transfection Reagent (Invitrogen) according to the manufacturer's protocol.

2.5. Assessment of Cell Viability. The 3-(4,5-dimethyithiazol2-yl)-2,5-diphenyl-tetrazolium bromide (MTT) assay was used to determine cellular mitochondrial dehydrogenase activity in primary cortical cells $\left(2 \times 10^{4} / \mathrm{ml}\right)$ cultured in 96well plates. Dark blue formazan crystals formed in intact cells which were solubilized with dimethyl sulfoxide (DMSO). The absorbance was measured at $490 \mathrm{~nm}$ with a microplate reader (Thermo Fisher Scientific, Waltham, MA, USA). Results were expressed as the percentage of MTT reduced, and data were normalized to the absorbance of control cells, considered $100 \%$.

2.6. Measurement of Apoptosis. Primary cortical cells $\left(9 \times 10^{5} / \mathrm{ml}\right)$ cultured in 6 -well plates were harvested and processed in accordance with the procedure of the Annexin V-FITC Apoptosis Detection Kit (BD Biosciences). Finally, flow cytometry (Epics-XL, Beckman Coulter, USA) was performed to quantify apoptosis. The results are expressed as percent of the control value.

2.7. Measurement of Antioxidative Enzyme Activity and Malondialdehyde Level. In vivo cerebral cortical tissue was dissected and homogenized, and in vitro cells $\left(4 \times 10^{5} / \mathrm{ml}\right)$ were harvested and lysed using ultrasound, then centrifuged at $12,000 \mathrm{~g}$ for $5 \mathrm{~min}$ at $4^{\circ} \mathrm{C}$. The supernatants were collected, and the activities of superoxide dismutase (SOD) and glutathione peroxidase (GPx), as well as the malondialdehyde (MDA) concentration, were detected according to the manufacturer's assay kits (Nanjing Jiancheng Bio-Engineering Institute Co., Ltd.).

2.8. Assay of Intracellular Total ROS Levels. Intracellular ROS production in primary cells was assessed by measuring the fluorescence intensity of 2,7-dichlorodihydrofluorescein diacetate (DCFH-DA). After being reoxygenated for $20 \mathrm{~h}$, cells $\left(9 \times 10^{5} / \mathrm{ml}\right)$ were treated with $10 \mu \mathrm{M}$ DCFH-DA in PBS in the dark at $37^{\circ} \mathrm{C}$ for $30 \mathrm{~min}$, and then washed with PBS to remove excess dye. The level of fluorescence intensity was immediately evaluated at (excitation) $488 \mathrm{~nm}$ and (emission) $525 \mathrm{~nm}$ with a microplate reader (BioTek Synergy H4, USA).

2.9. Measurement of Intracellular cAMP Concentration. Primary cortical neurons were seeded in 6-well plates, and cells $\left(1 \times 10^{6} / \mathrm{ml}\right)$ were subjected to $\mathrm{OGD} / \mathrm{R}$, then harvested by centrifugation at $1000 \mathrm{~g}$ for $10 \mathrm{~min}$ at $4^{\circ} \mathrm{C}$. Cerebral cortical tissue and cells were homogenized in $50 \mathrm{mmol} / \mathrm{l}$ acetic acid buffer ( $\mathrm{pH}$ 4.75) and lysed by sonication. The homogenate was centrifuged at $3000 \mathrm{~g}$ for $15 \mathrm{~min}$ at $4^{\circ} \mathrm{C}$. cAMP levels in the supernatant were determined using a ${ }^{125}$ I-radioimmuno- assay according to the RIA kit instructions (Nuclear Medicine Laboratory of Shanghai University of Traditional Chinese Medicine, Shanghai, PR China).

2.10. Real-Time PCR. The expression levels of AK046177 and miR-134 were analyzed by real-time PCR in both brains and cell cultures after I/R or OGD/R. Total RNA was isolated from $30 \mathrm{mg}$ cerebral cortical tissue or cells $\left(1 \times 10^{6} / \mathrm{ml}\right)$ seeded on 6-well plates with TRIzol Reagent (Invitrogen) [29], and reverse transcribed into cDNA using miScript Reverse Transcription Kit (Takara, China). Predesigned PCR primer/probes of miR-134 were obtained from GenePharma (Shanghai, China) for AK046177 and miRNA-134, with $\beta$-actin and U6 small nuclear RNA (U6) used as an internal control. Quantitative PCR was conducted as previously described using the TaqMan Assay Kit (Applied Biosystems) [30]. The relative expression levels of AK046177 and miRNA-134 were calculated using the $2^{-\triangle \Delta C T}$ method [31].

2.11. Observation of Mitochondrial Morphology. Mitochondrial ultrastructure was analyzed using a transmission electron microscope (TEM) (Olympus, Tokyo, Japan). Primary cortical neurons were seeded in a 6-well plate and treated as described as above. After reperfusion, cells $\left(9 \times 10^{5} / \mathrm{ml}\right)$ were harvested and fixed in $2.5 \%(v / v)$ glutaraldehyde, then collected into a centrifuge tube using a scraper. Samples were fixed in osmic acid, dehydrated by the gradual addition of ethanol, and embedded in epoxy resin. Embedded samples were sectioned into ultraslices, stained with uranyl acetate and lead citrate, and then observed by TEM.

2.12. Measurement of Cell Respiration. Oxygen consumption rate (OCR) was measured using high-resolution respirometry in a 2-channel titration injection respirometer at $37^{\circ} \mathrm{C}$ (Oxygraph-2k, Oroboros, Innsbruck, Austria), as previously described by Pesta and Gnaiger [32]. Briefly, after reperfusion, cells $\left(1 \times 10^{6} / \mathrm{ml}\right)$ were harvested and placed in $A$ and $\mathrm{B}$ pools with DMEM. Three readings were taken after the addition of each of the following mitochondrial inhibitors, prior to injection with the subsequent inhibitors. Inhibitors used were oligomycin $(2 \mathrm{mg} / \mathrm{ml})$, carbonyl cyanide chlorophenylhydrazine (CCCP; $10 \mathrm{mM})$, and rotenone $(2 \mathrm{mM})$. OCR was automatically calculated and recorded by a sensor cartridge and Oroboros software.

2.13. Western Blot Analysis. Cerebral cortex tissues or cell cultures $\left(1 \times 10^{6} / \mathrm{ml}\right)$ were homogenized with lysis buffer and centrifuged at $12,000 \mathrm{~g}$ for $15 \mathrm{~min}$. Forty micrograms of protein from each sample was separated on a SDS/10 and $15 \%$ polyacrylamide gel and then transferred to a polyvinylidene difluoride (PVDF) membrane (Millipore, IPVH00010, Bedford, MA, USA). After being blocked with $1 \%$ bovine serum albumin for $120 \mathrm{~min}$ at $20^{\circ} \mathrm{C} \pm 2^{\circ} \mathrm{C}$, membranes were incubated with anti-Nrf2 (1:1000; SAB4501984; SigmaAldrich), anti-Bcl-2 (1:1000; SAB4500003; Sigma-Aldrich), anti-Bax (1:1000; SAB4502546; Sigma-Aldrich), anticaspase 3 (1:500; ab32042; Abcam), anti-CREB (1:1000; SAB4500441; Sigma-Aldrich), anti-phospho-CREB (pSer ${ }^{133}$; 


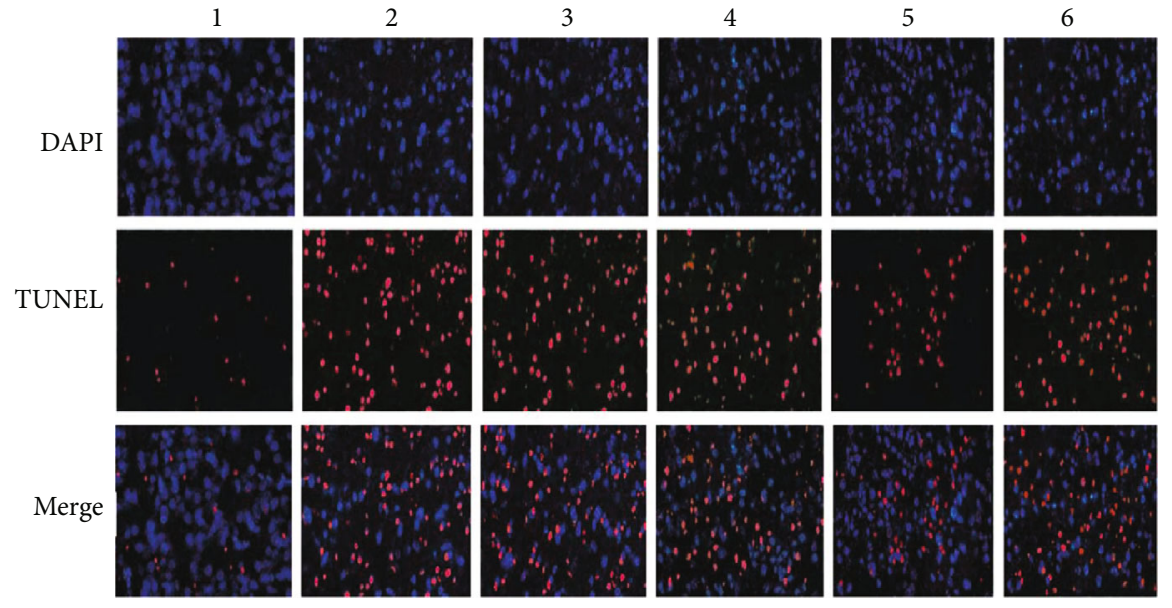

(a)
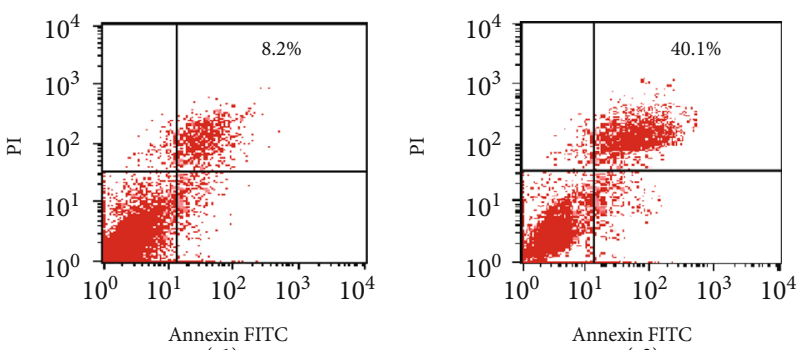

(c1)
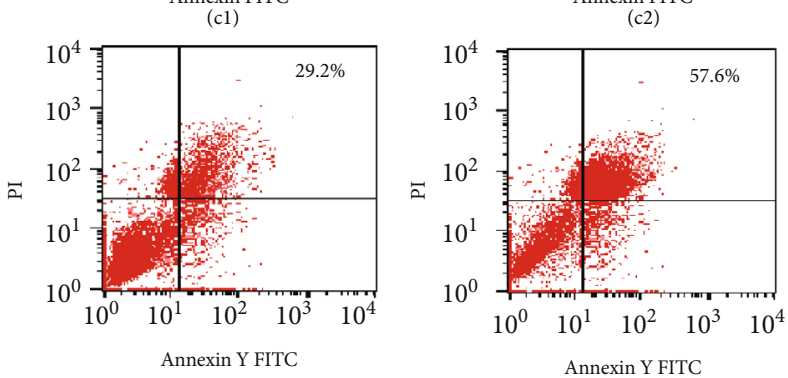

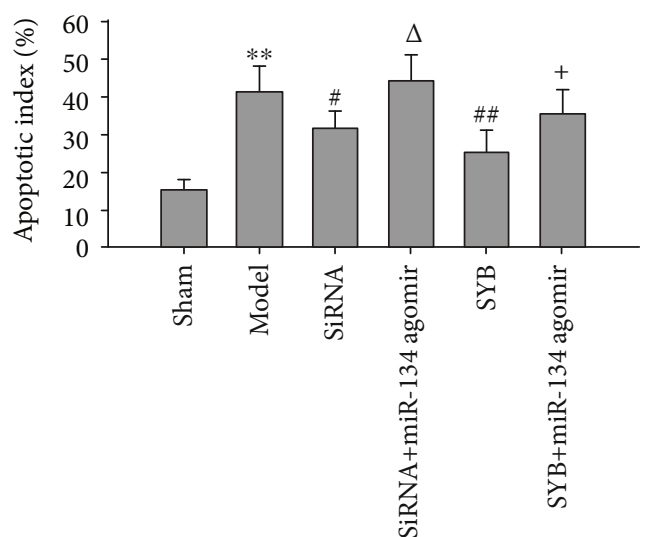

(b)
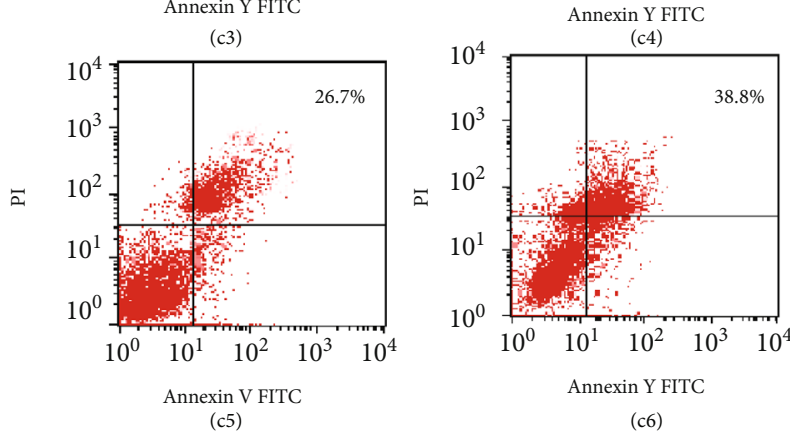

(c)

Figure 2: Continued. 


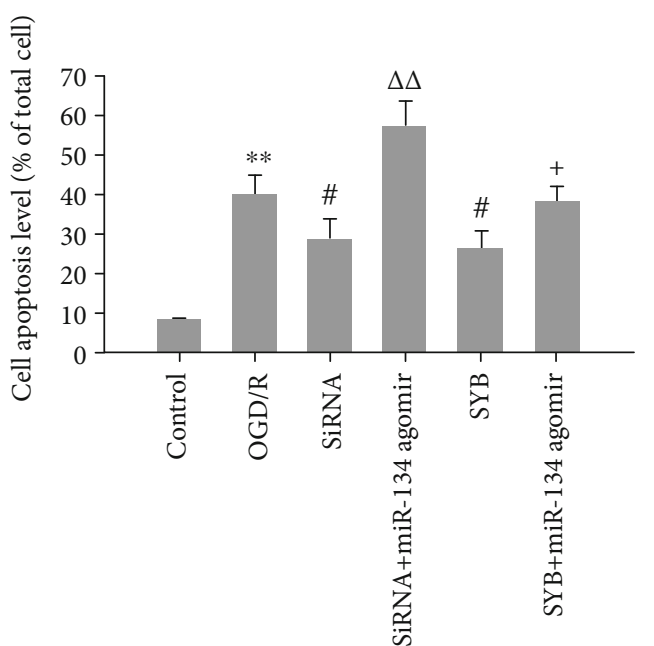

(d)

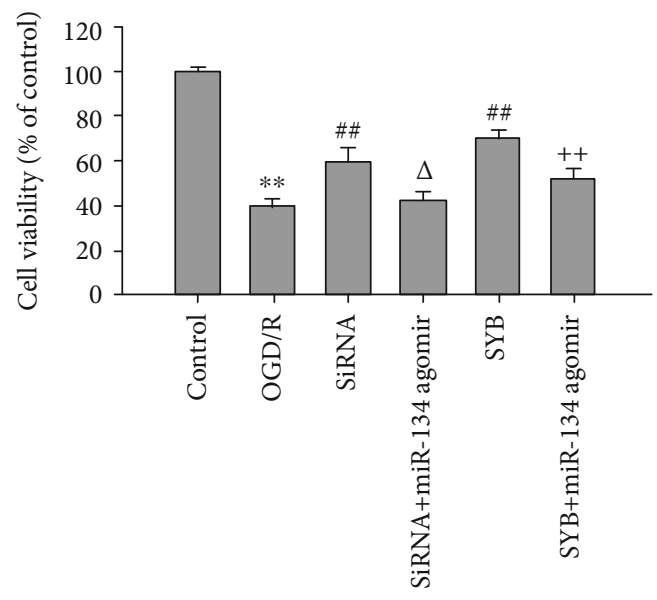

(e)

FIgURE 2: Effect of safflor yellow B on TUNEL-positive cells, cell viability, and apoptosis. Rats were divided into six groups: sham, ischemia/reperfusion (I/R), AK046177 siRNA, AK046177 siRNA+miR-134 agomir, SYB, and SYB+miR-134 agomir. (a) Representative images showing TUNEL-positive cells of cerebral cortex in different groups $(\times 200$ magnification; 1, 2, 3, 4, 5, and 6 represent sham, ischemia/reperfusion (I/R), AK046177 siRNA, AK046177 siRNA+miR-134 agomir, SYB, and SYB+miR-134 agomir, respectively). (b) Apoptotic (TUNEL-positive) cells were detected, $\mathrm{AI}=$ (number of apoptotic cells/total cell number counted) $\times 100 \%(n=3)$. Primary fetal cortical cells were seeded in 96-well and 6-well plates and divided into six groups: control, OGD/R, AK046177 siRNA, AK046177 siRNA +miR-134 agomir, SYB, and SYB+miR-134 agomir. Apart from the control group, all cells in the other groups were cultured in glucosefree DMEM and hypoxic conditions $\left(1 \% \mathrm{O}_{2} / 94 \% \mathrm{~N}_{2} / 5 \% \mathrm{CO}_{2}\right)$ at $37^{\circ} \mathrm{C}$ for 4 h. Thereafter, all groups' media were replaced with normal DMEM, and culturing continued for $20 \mathrm{~h}$ of reoxygenation under normoxic conditions $\left(95 \%\right.$ air $\left./ 5 \% \mathrm{CO}_{2}\right)$. The cells were pretreated with SYB, AK046177, and miR-134 agomir before being exposed to OGD/R. Cell viability was detected by the MTT method. Cell apoptosis was analyzed using flow cytometry. (c1)-(c6) represent control, OGD/R, AK046177 siRNA, AK046177 siRNA+miR-134 agomir, SYB, and SYB+miR-134 agomir, respectively. (d) represents cell apoptosis $(n=3)$. (e) represents cell viability $(n=8)$. Data are presented as mean \pm SD. One-way ANOVA test was used to determine statistical significance. ${ }^{* *} P<0.01 v$ s. the sham group or the control group, ${ }^{\#} P<0.05$ or ${ }^{\# \#} P<0.01 v$ s. the I/R group or the OGD/R group, ${ }^{\Delta} P<0.05$ or ${ }^{\Delta \Delta} P<0.01 v$ s. the AK046177 siRNA group, and ${ }^{+} P<0.05$ or ${ }^{++} P<0.01 v$. the SYB group.

1:1000; C9102; Sigma-Aldrich), or anti-Nox4 (1:500; ab109225; Abcam) overnight at $4^{\circ} \mathrm{C}$ and washed with TBST $(3 \times 15 \mathrm{~min})$. The corresponding horseradish peroxidase(HRP-) conjugated secondary antibody was added and left for $50-60 \mathrm{~min}$ at $20^{\circ} \mathrm{C} \pm 2^{\circ} \mathrm{C}$, followed by washing with TBST $(3 \times 10 \mathrm{~min})$. Protein bands were visualized using a chemiluminescence reagent (ECL kit; Amersham Corporation, Arlington Heights, CA, USA). The relative density of the protein bands was quantified by densitometry using an Image Acquiring and Analysis System (Leica Com., Germany). $\beta$-Actin was used to normalize protein loading. ERK1/2 phosphorylation was calculated as the ratio of normalized arbitrary units (a.u.) of (phosphorylated ERK1/2)/(total ERK1/2).

2.14. Determination of NADPH Oxidase Activity. NADPH oxidase activity was assayed using an Amplite ${ }^{\mathrm{TM}}$ Fluorime NADPH Assay Kit (AAT Bioquest, USA) according to the manufacturer's instructions. Primary cortical neurons were seeded in 24-well plates and treated. Rats were treated in vivo as described above. Cells $\left(4 \times 10^{5} / \mathrm{ml}\right)$ and tissues were lysed or homogenized, then centrifuged. The supernatant and NADPH oxidase reaction mixture were incubated at $37^{\circ} \mathrm{C}$ for $2 \mathrm{~h}$. The increase in fluorescence was monitored using a microplate reader (BioTek Synergy H4, USA) at excitation and emission wavelengths of 540 and $590 \mathrm{~nm}$, respectively.

2.15. Statistical Analysis. Data are presented as mean \pm standard deviation (SD). The statistical analysis of the results was performed via one-way analysis of variance (ANOVA) followed by Student's $t$-test. All analyses were performed using SPSS 10.0 software (SPSS, Inc., San Rafael, CA, USA). Probabilities lower than $5 \%(P<0.05)$ were considered statistically significant.

\section{Results}

3.1. Effect of SYB on Neurological Deficit Score, Infarct Volume, and Motor Function. As shown in Figures 1(b)1(e), I/R significantly enhanced neurological deficit score and infarcted area and decreased motor scores compared to shams $(P<0.05$ and $P<0.01)$. Intraventricular injection of AK046177 siRNA or intravenous injection of SYB was able to significantly decrease neurological deficit scores and infarct volume and enhance motor scores $(P<0.05$ and $P<0.01$, respectively). In addition, miR-134 agomir was capable of reversing the improvement in neurological deficit score, infarct volume, and motor scores of SYB and AK046177 siRNA $(P<0.05$ and $P<0.01$, respectively). 

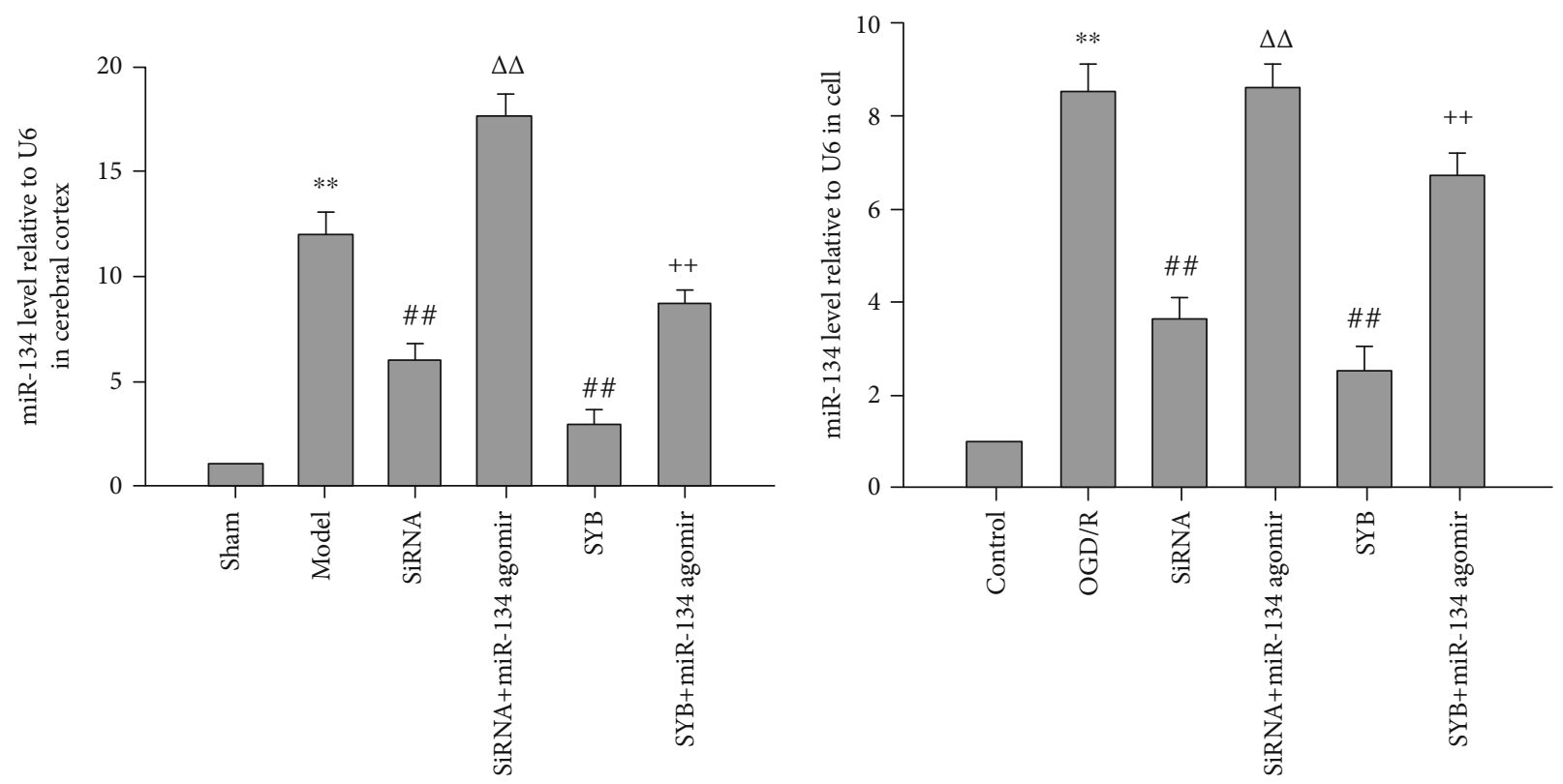

(a)
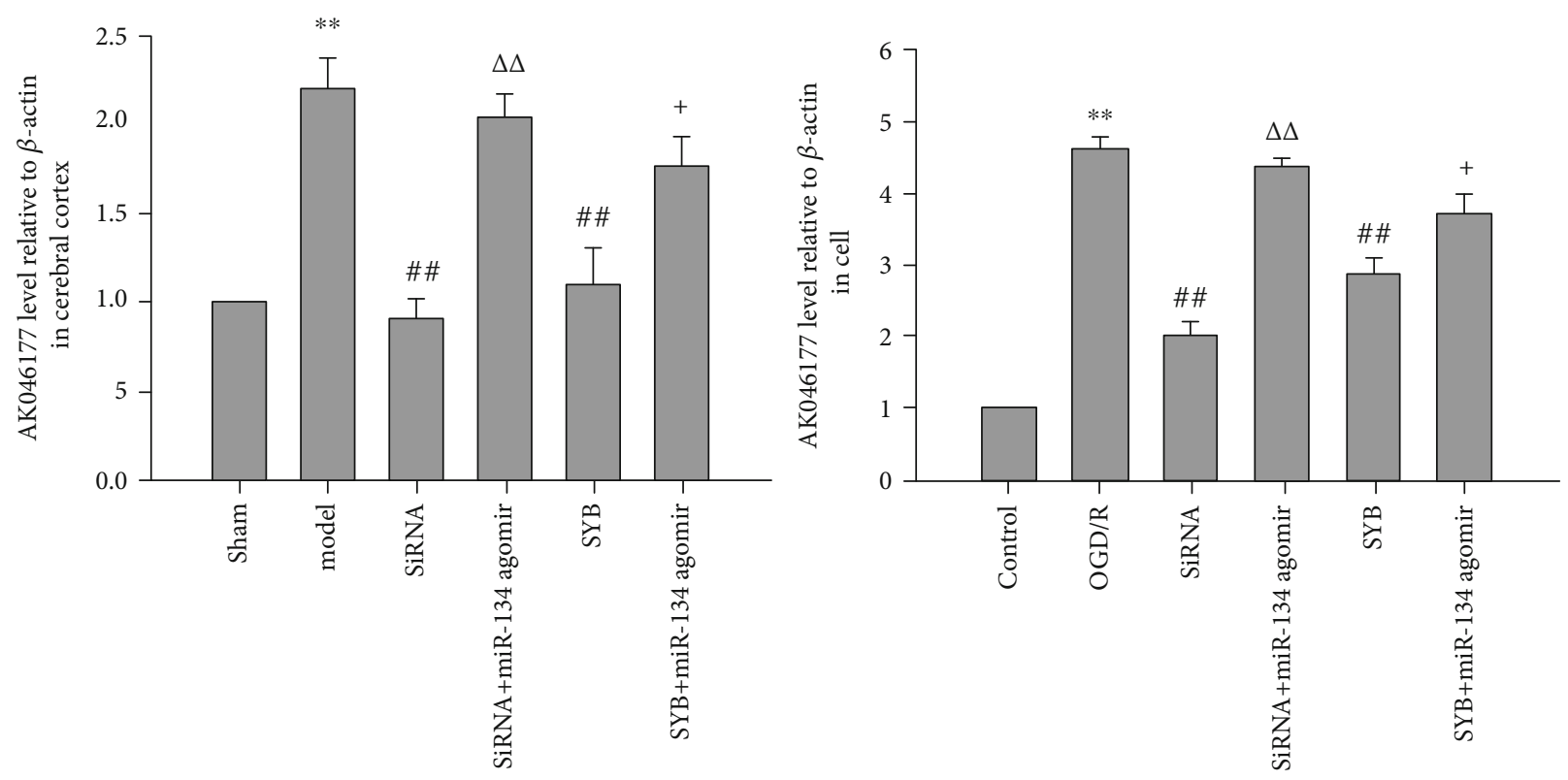

(b)

FIgure 3: Continued. 


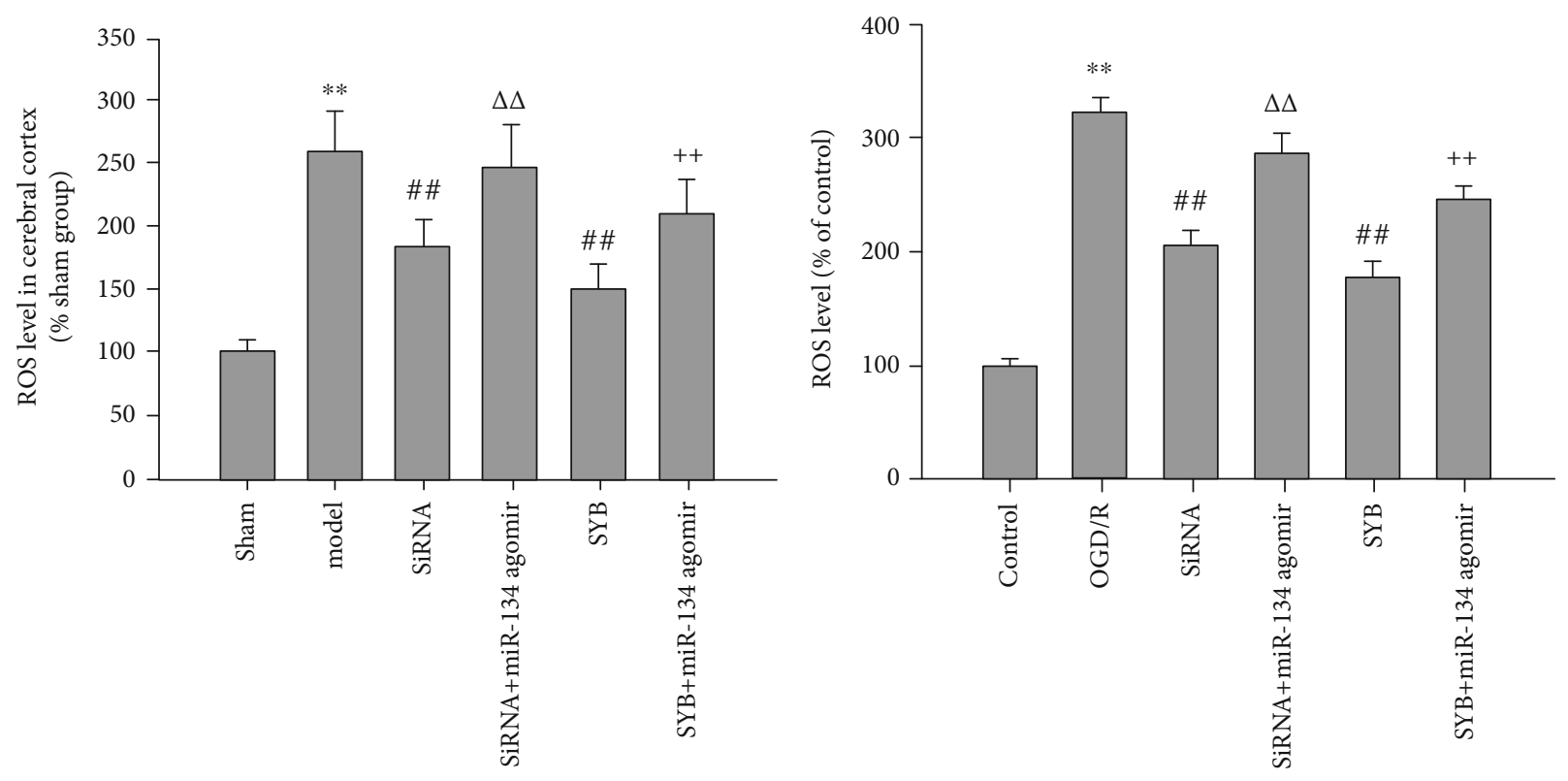

(c)

FIGURE 3: Effect of safflor yellow B on ROS level and the expression of AK046177 and miR-134. Rats and primary fetal cortical cells were used to establish the I/R model and the OGD/R model, respectively. After I/R or OGD/R for $24 \mathrm{~h}$, the total RNA of every group was isolated and then reverse transcribed into cDNA. The expression levels of AK046177 and miR-134 were measured by real-time PCR. (a1), (a2), (b1), and (b2) represent miR-134 and AK046177 expression levels in the cerebral cortex and primary fetal cortical cells, respectively. ROS generation was measured according to the procedure of the assay kit provided by the manufacturer. (c1) and (c2) represent ROS levels in the cerebral cortex and the primary fetal cortical cells, respectively. Data are presented as mean $\pm \mathrm{SD}$ ( $n=8$ in tissues; $n=3$ in cells). One-way ANOVA test was used to determine statistical significance. ${ }^{* *} P<0.01 v s$. the sham group or the control group, ${ }^{\# \#} P<0.01 v s$. the I/R group or the OGD/R group, ${ }^{\Delta \Delta} P<0.01 v s$. the AK046177 siRNA group, and ${ }^{+} P<0.05$ or ${ }^{++} P<0.01 v s$. the SYB group.

3.2. Effect of SYB on Cell Viability and Apoptosis. I/R and $\mathrm{OGD} / \mathrm{R}$ produced significant increases in the number of apoptotic cells in vivo $(P<0.01$; Figures 2(a), 2(b), 2(c1), $2(\mathrm{c} 2)$, and 2(d)), as well as significant reductions in cell viability in vitro $(P<0.01$; Figure $2(\mathrm{e}))$. This effect was significantly alleviated by AK046177 siRNA and SYB $(P<0.05$ and $P<0.01$, respectively; Figures 2(a), 2(b), 2(c3), 2(c5), 2(d), and 2(e)). miR-134 agomir inhibited the protective effect of AK046177 siRNA and SYB and then aggravated I/R- and OGD/R-mediated cell death and damage $(P<0.05$ and $P<0.01$, respectively; Figures 2(a), 2(b), 2(c4), 2(c6), $2(\mathrm{~d})$, and $2(\mathrm{e}))$.

3.3. Effect of SYB on the Intracellular Expression of AK046177 and miR-134 and cAMP Content. As shown in Figures 3(a1), 3(a2), 3(b1), and 3(b2), I/R and OGD/R significantly increased the expression levels of $A K 046177$ and miR-134 $(P<0.01)$, which were decreased by the addition of AK046177 siRNA $(P<0.01)$. SYB effectively attenuated $\mathrm{I} / \mathrm{R}$ and $\mathrm{OGD} / \mathrm{R}$ induced increases in the expressions of $A K 046177$ and miR-134 $(P<0.01)$, while the inclusion of miR-134 agomir reversed the SYB-induced attenuation $(P<0.05$ and $P<0.01)$. Figures $1(\mathrm{f})$ and $1(\mathrm{~g})$ demonstrate the significant reduction in intracellular cAMP levels caused by I/R and OGD/R; these reductions were further aggravated by miR-134 agomir $(P<0.05$ and $P<0.01$, respectively). Following treatment with AK046177 siRNA and SYB, intracellular cAMP levels rose significantly in both the cerebral cortex and the primary cultures $(P<0.05$ and $P<0.01)$. miR-134 agomir significantly reduced the SYB- and AK046177 siRNA-induced increase in intracellular cAMP levels $(P<0.05)$.

3.4. Effect of SYB on SOD and GPX Activities and MDA Levels. As shown in Figures 4(a1), 4(a2), 4(b1), and 4(b2), the activities of SOD and GPx following I/R and OGD/R were reduced $(P<0.01)$, and MDA levels were increased $(P<0.01$; Figures $4(\mathrm{c} 1)$ and $4(\mathrm{c} 2))$ relative to those of the control group. Compared to the I/R or OGD/R groups, SYB significantly increased the activities of SOD and GPx in vitro and in vivo $(P<0.01)$. AK046177 siRNA promoted GPx activity in cells in a manner greater than the effect on SOD activity in vitro and in vivo and GPx activity in vitro $(P<0.01)$. SYB and AK046177 siRNA caused a significant reduction in MDA levels $(P<0.01)$. The effects of SYB and AK046177 siRNA were significantly suppressed by miR-134 agomir $(P<0.05$ and $P<0.01$, respectively).

3.5. Effect of SYB on NADPH Oxidase Activity, Nox4 Expression, and ROS Generation. As shown in Figures 5(a1) and 5(a2), compared to the control group, I/R and OGD/R significantly increased NADPH oxidase activity $(P<0.01)$. Compared to the I/R or OGD/R groups, AK046177 siRNA and SYB were capable of significantly abolishing NADPH oxidase activity $(P<0.01)$. After treatment with miR-134 agomir, NADPH oxidase activity in the cerebral cortex and 

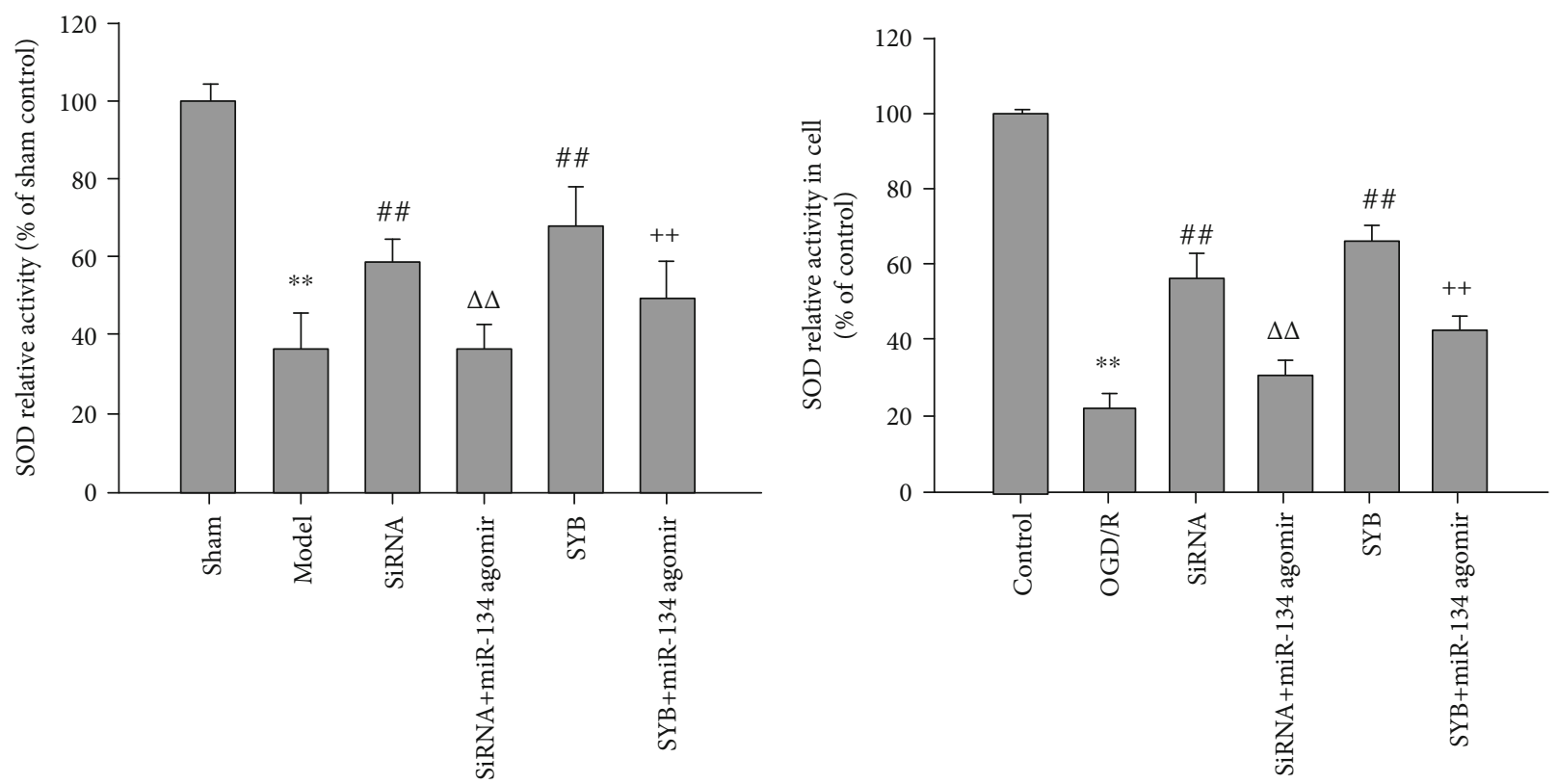

(a)
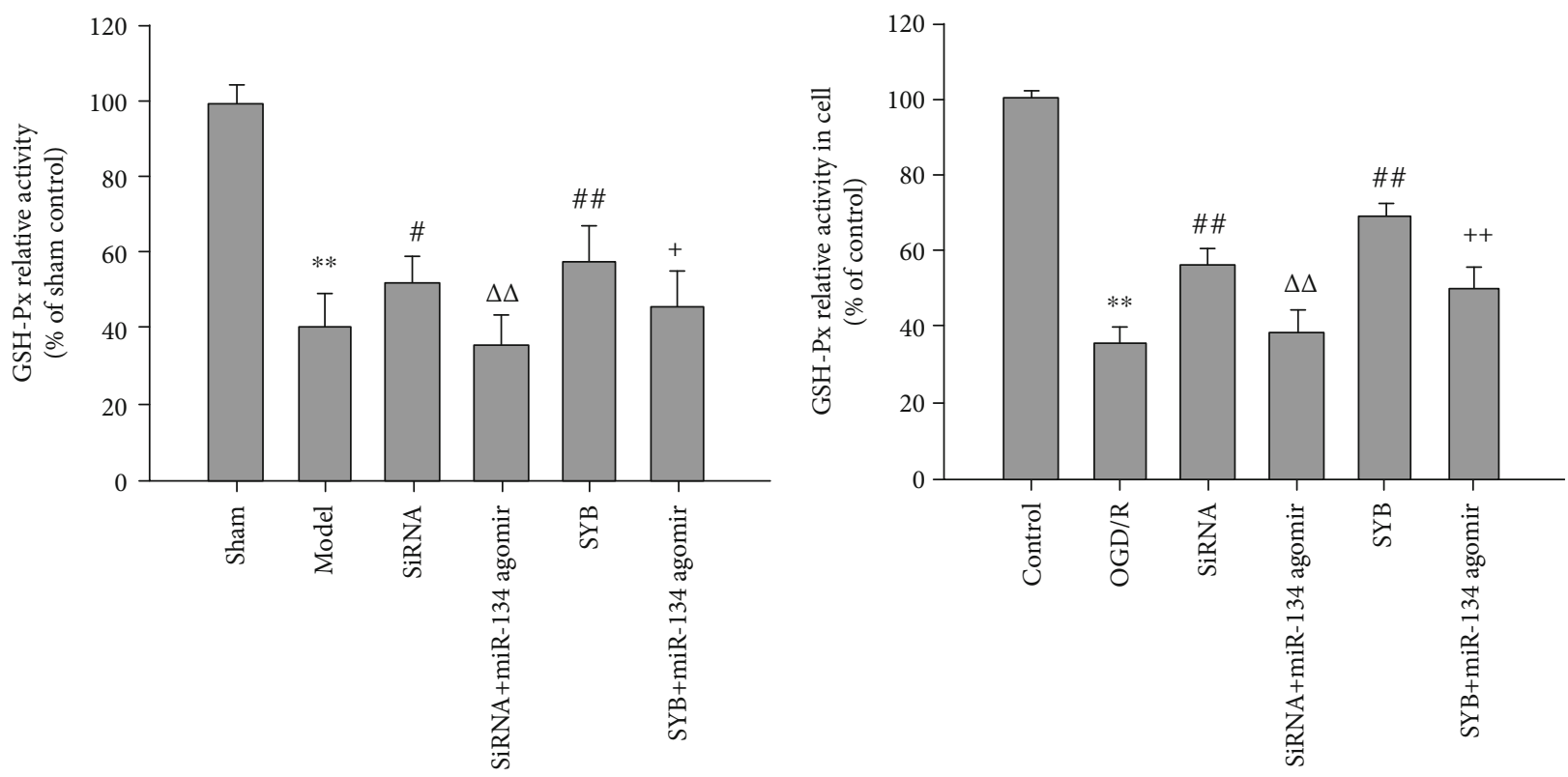

(b)

FIgURE 4: Continued. 

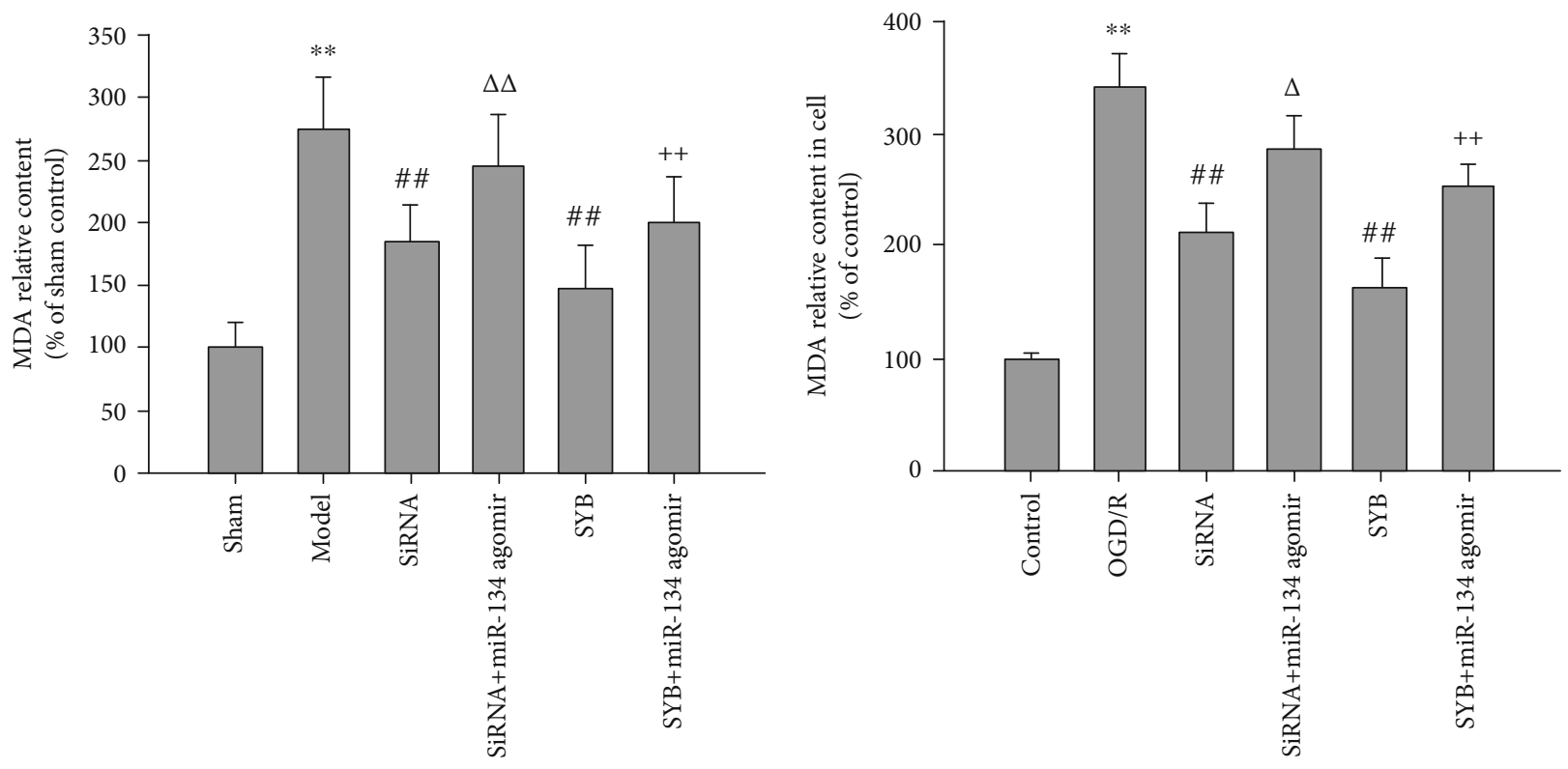

(c)

FIGURE 4: Effect of safflor yellow B on antioxidant enzyme activity and MDA level. Rats and primary fetal cortical cells were used to establish the I/R model and the OGD/R model, respectively. After I/R for $24 \mathrm{~h}$, the cerebral cortex and cells from different groups were collected. The activities of SOD and GSH-Px and the MDA levels in tissues and cells were determined with the spectrophotometrical method according to the procedure described by the assay kit. (a1), (b1), and (c1) represent the activities of SOD and GSH-Px and the MDA level in cerebral cortex tissue. (a2), (b2), and (c2) represent the activities of SOD and GSH-Px and the MDA level in cells. Data are presented as mean \pm SD $(n=8$ in brain tissues, or $n=3$ in cells). One-way ANOVA test was used to determine statistical significance. ${ }^{* *} P<0.01 v s$. the sham group or the control group, ${ }^{\#} P<0.05$ or ${ }^{\# \#} P<0.01 v s$. the I/R group or the OGD/R group, ${ }^{\Delta} P<0.05$ or ${ }^{\Delta \Delta} P<0.01 v s$. the AK046177 siRNA group, and ${ }^{+} P<0.05$ or ${ }^{++} P<0.01 v$ s. the SYB group.

primary neuronal cultures showed a significant increase relative to the SYB and AK046177 siRNA groups $(P<0.05$ and $P<0.01$, respectively).

Nox4 directly reflects NADPH oxidase activity. Our data showed that $\mathrm{I} / \mathrm{R}$ and $\mathrm{OGD} / \mathrm{R}$ significantly promoted Nox4 expression relative to the sham and control groups $(P<0.01)$. Both SYB and AK046177 siRNA were able to significantly suppress Nox4 expression, an effect reversed by miR-134 agomir $(P<0.01$ and $P<0.05$; Figures $5(\mathrm{~b} 1)$, 5(b2), 5(c1), and 5(c2)).

ROS levels in cells exposed to OGD/R and cerebral cortex of rats induced by $\mathrm{I} / \mathrm{R}$ were higher than those of the control group and the sham group $(P<0.01$; Figures $3(\mathrm{c} 1)$ and $3(\mathrm{c} 2))$. This effect was significantly mitigated by AK046177 siRNA and SYB, which also decreased ROS generation relative to the OGD/R group and the sham group $(P<0.01)$. The inhibitory effects of SYB and AK046177 siRNA on ROS generation were significantly ameliorated by miR-134 agomir in vitro $(P<0.01)$.

3.6. Effect of SYB on the Expressions of Nrf2, CREB, pCREB, Caspase3, Bcl-2, and Bax. As seen in Figures 6(a1)-6(e2), the ratio of $\mathrm{Bax} / \mathrm{Bcl}-2$ and caspase 3 expression in the $\mathrm{I} / \mathrm{R}$ and $\mathrm{OGD} / \mathrm{R}$ groups were higher than those in the sham or control groups $(P<0.01)$. AK046177 siRNA and SYB significantly increased $\mathrm{Nrf} 2$ expression and the ratio of pCREB/CREB and decreased caspase 3 expression and the ratio of $\mathrm{Bax} / \mathrm{Bcl}-2$, relative to the $\mathrm{I} / \mathrm{R}$ and $\mathrm{OGD} / \mathrm{R}$ groups
$(P<0.01$ and $P<0.05)$. miR-134 agomir reversed these effects, and increased the ratio of $\mathrm{Bax} / \mathrm{Bcl}-2$ and caspase 3 expression, and reduced the ratio of $\mathrm{pCREB} / \mathrm{CREB}$ and Nrf2 expression, relative to the AK046177 siRNA and SYB groups $(P<0.01$ and $P<0.05)$.

3.7. Effect of SYB on Mitochondrial Structure and Cell Respiration. In the control group, the mitochondria were diffused throughout the cell, with an oval or rod-like shape and normal structure (Figure 7(a1)). In OGD/R-treated cells, mitochondria exhibited pathological changes including irregular and swollen shapes, mitochondrial ridge fault, and vesicular mitochondrial clusters, especially adjacent to the cell nucleus (Figure 7(a2)). After treatment with SYB and AK046177 siRNA, an increased number of normal mitochondria were detected (Figures $7(\mathrm{a} 3)$ and $7(\mathrm{a} 5)$ ). miR-134 agomir in addition to SYB and AK046177 siRNA resulted in more irregular and swollen shapes, more mitochondrial cristae fracturing, and severe vacuolization within mitochondria compared to the SYB group (Figures 7(a4) and 7(a6)). Moreover, AK046177 siRNA and SYB were capable of effectively alleviating mitochondrial pathology induced by miR-134 agomir and OGD/R.

OGD/R markedly influenced cellular respiration and decreased the OCR (Figure 7(b)). The OCR in all groups was significantly reduced after treatment with oligomycin. Cells normally increase OCR in response to CCCP in order to maintain the proton gradient and mitochondrial function. 

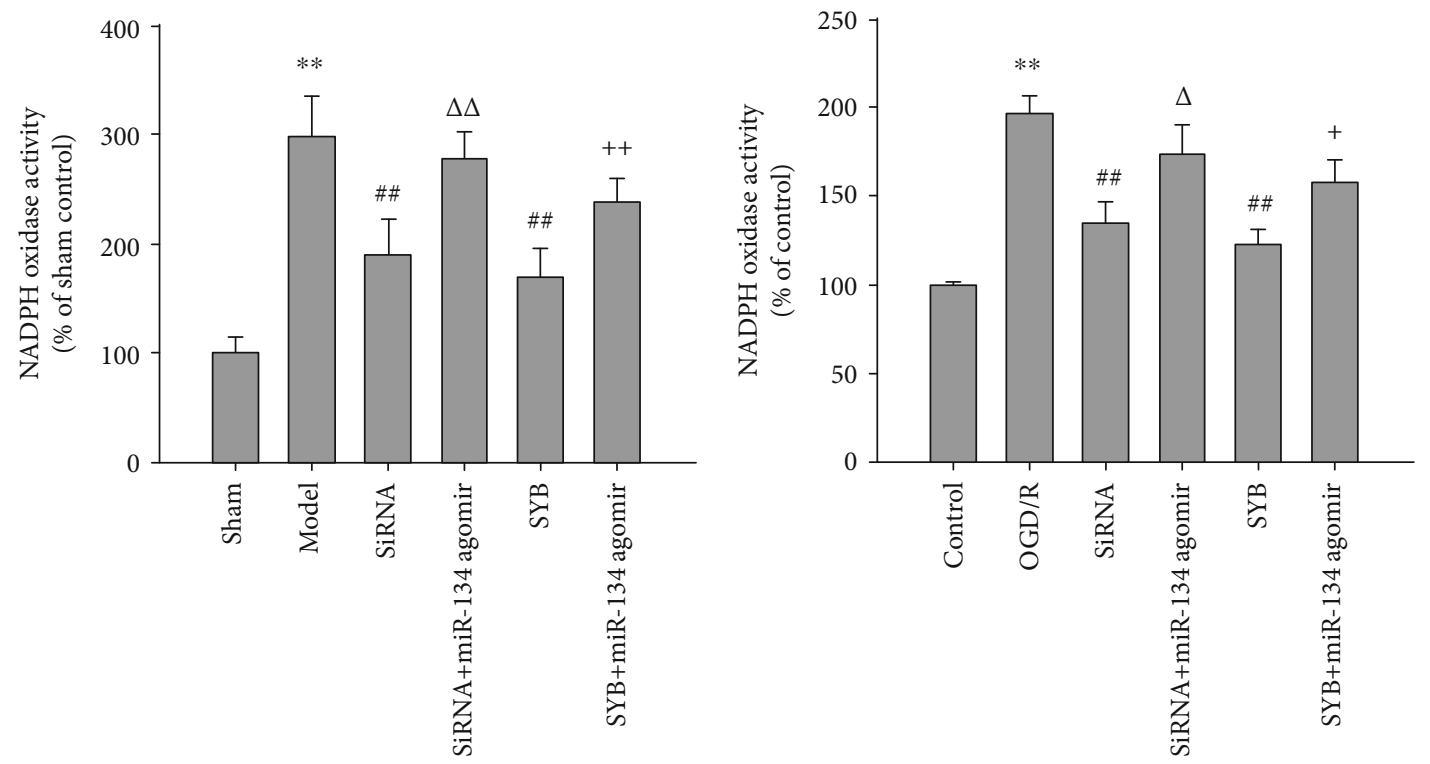

(a)
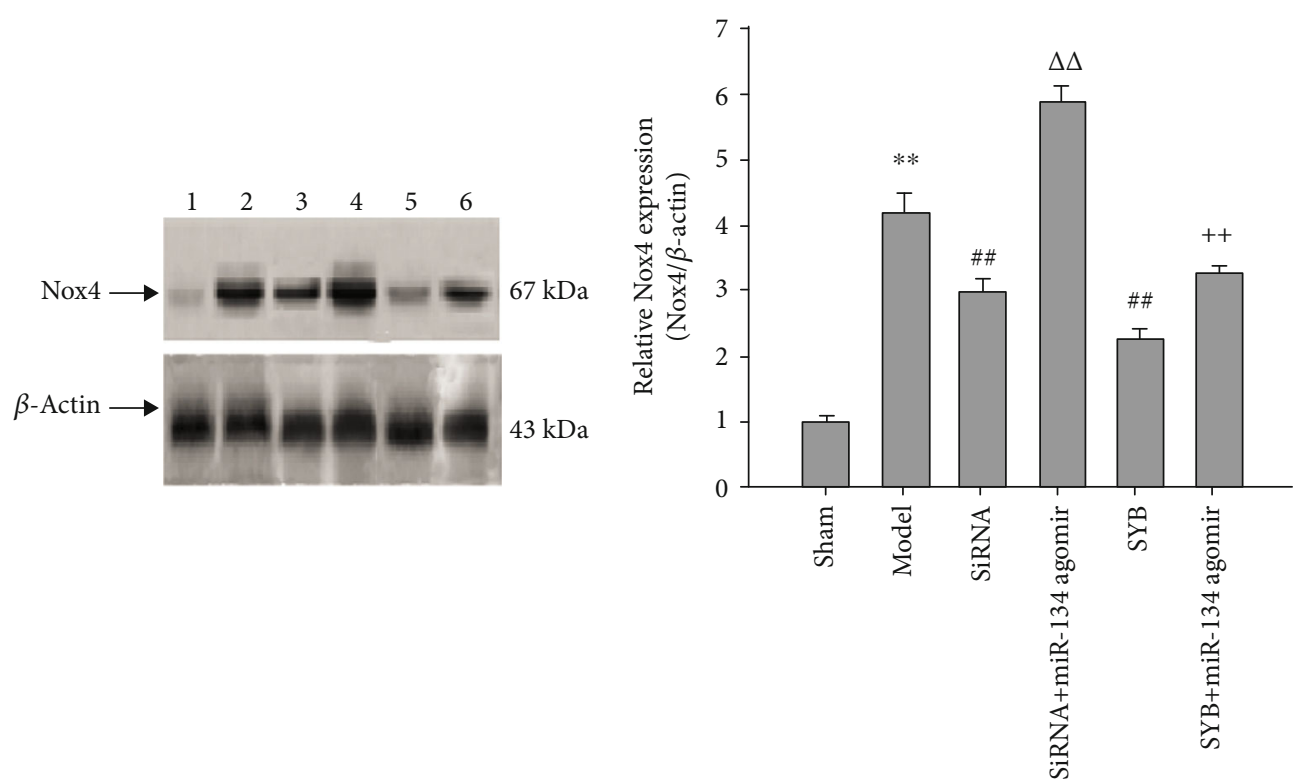

(b)

Figure 5: Continued. 

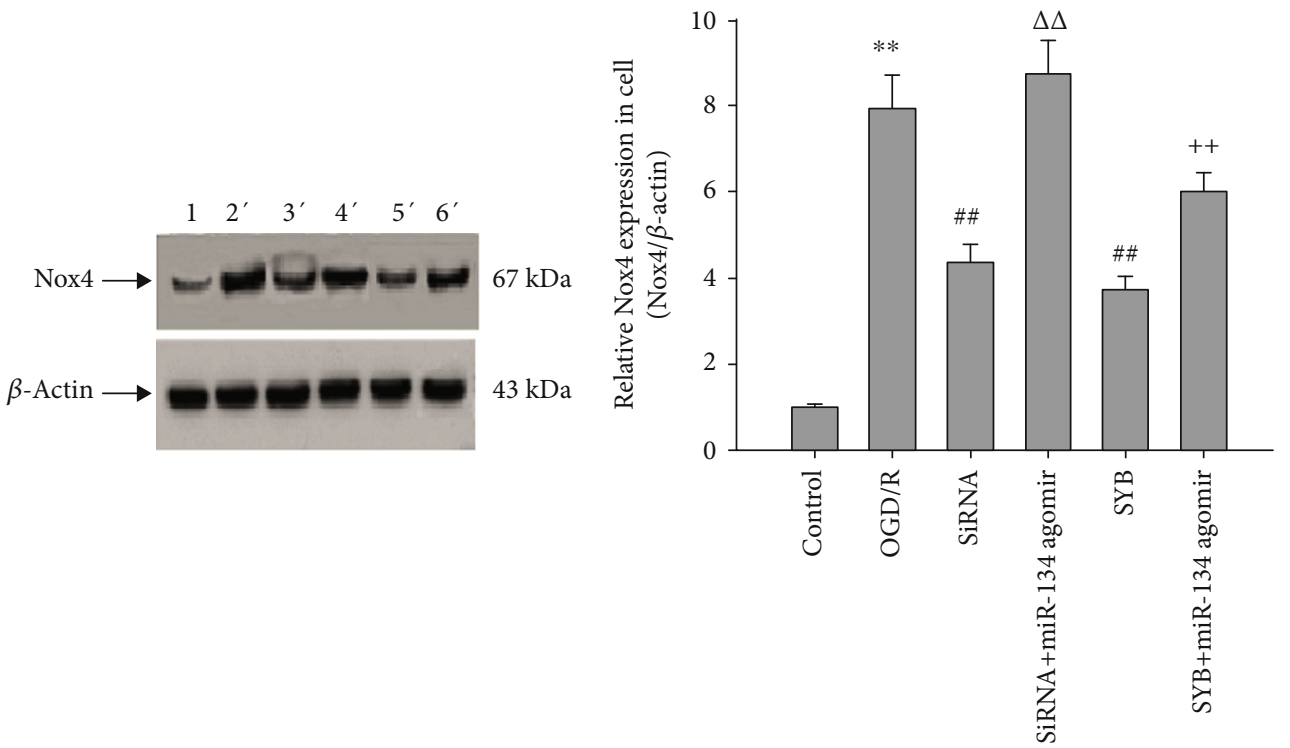

(c)

FIGURE 5: Effect of safflor yellow B on NADPH oxidase activity and Nox4 expression. Rats and primary fetal cortical cells were used to establish the I/R model and the OGD/R model, respectively. After I/R for $24 \mathrm{~h}$, the cerebral cortex and cells from different groups were collected. NADPH oxidase activity and Nox4 expression was observed using Western blot analysis. (a1) and (a2) represent the NADPH oxidase activity of the cerebral cortex and the primary fetal cortical cells, respectively. (b1) Representative western blots of the cerebral cortex are shown $(1,2,3,4,5$, and 6 represent sham, ischemia/reperfusion (I/R), AK046177 siRNA, AK046177 siRNA+miR-134 agomir, SYB, and SYB+miR-134 agomir, respectively). (b2) represents Nox4 expression in the cerebral cortex. (c1) Representative western blots of primary fetal cortical cells are shown $\left(1^{\prime}, 2^{\prime}, 3^{\prime}, 4^{\prime}, 5^{\prime}\right.$, and $6^{\prime}$ represent control, OGD/R, AK046177 siRNA, AK046177 siRNA+miR-134 agomir, SYB, and SYB+miR-134 agomir, respectively). (c2) represent Nox4 expression in cells. Data are presented as mean \pm SD $(n=8$ in brain tissues, or $n=3$ in cells). One-way ANOVA test was used to determine statistical significance. ${ }^{* *} P<0.01 v s$. the sham group or the control group, ${ }^{\# \#} P<0.01 v$ s. the I/R group or the OGD/R group, ${ }^{\Delta} P<0.05$ or ${ }^{\Delta \Delta} P<0.01 v$ s. the AK046177 siRNA group, and ${ }^{+} P<0.05$ or ${ }^{++} P<0.01$ vs. the SYB group.

However, cells exposed to OGD/R had a smaller increase in OCR after CCCP treatment compared to controls, suggesting that these cells have impaired respiratory capacity. Administration of rotenone, a complex I inhibitor, also inhibited OCR. These data suggest that OGD/R results in the loss of mitochondrial oxidative phosphorylation (OXPHOS) function in primary neurons. Furthermore, AK046177 siRNA and SYB enhanced OCR after CCCP treatment relative to the OGD/R group, suggesting that AK046177 siRNA and SYB were able to improve mitochondrial respiratory capacity. Under OGD/R conditions, miR-134 agomir significantly reduced the OCR increase induced by AK046177 siRNA and SYB and amplified OGD/R-mediated cell injury, resulting in much weaker respiratory capacity.

\section{Discussion}

lncRNAs may act to upregulate biological processes in various disease states by directly or indirectly interacting with mRNA of the target gene, leading to the modulation of apoptosis and invasion and modification of chromatin [18-20].

MicroRNAs are a type of small noncoding RNA involved in various diseases via their interaction with the mRNA of target genes, which leads to the destabilization and degradation of mRNA [33-35]. Di et al. have shown that a variety of miRNAs are involved in cerebral $I / R$ injury [36]. The brain-specific miR-134 was reported to be differentially expressed in tissue subjected to MCAO and I/R injury [37]. Previous studies had shown that lncRNAs influenced ischemia-mediated tissue damage via regulation of microRNAs [38-40]. In the present study, we demonstrated that $\mathrm{I} / \mathrm{R}$ and $\mathrm{OGD} / \mathrm{R}$ increased the expression levels of miR-134 in the cerebral cortex or primary neuronal cultures and resulted in neuronal damage accompanied by the upregulation of AK046177. Inhibition of AK046177 expression could significantly reduce the expression of miR-134, thereby alleviating I/R- or OGD/R-mediated neuronal injury.

Wang et al.'s studies have shown that SYB was able to effectively inhibit ischemia-induced brain injury [24]. However, the relationship between miR-134 or AK046177 and SYB in cerebral ischemia was unknown. These data confirm that SYB decreases the expression levels of miR-134 and AK046177 and reduces I/R- and OGD/R-induced damage.

Cyclic AMP is an important second messenger involved in many biochemical processes through its ability to regulate protein kinase activity. It is involved in diverse processes such as platelet activation, thrombus formation, inflammatory response, and oxidative stress responses [41-43]. Elevation of intracellular cAMP levels can change the antioxidant capacity of the cells by regulating the phosphorylation of CREB [44]. Thus, CREB has important influences in various diseases $[45,46]$. Substantial evidence indicates that CREB plays critical roles in the neuronal responses to ischemia, such that activation and overexpression of CREB significantly 

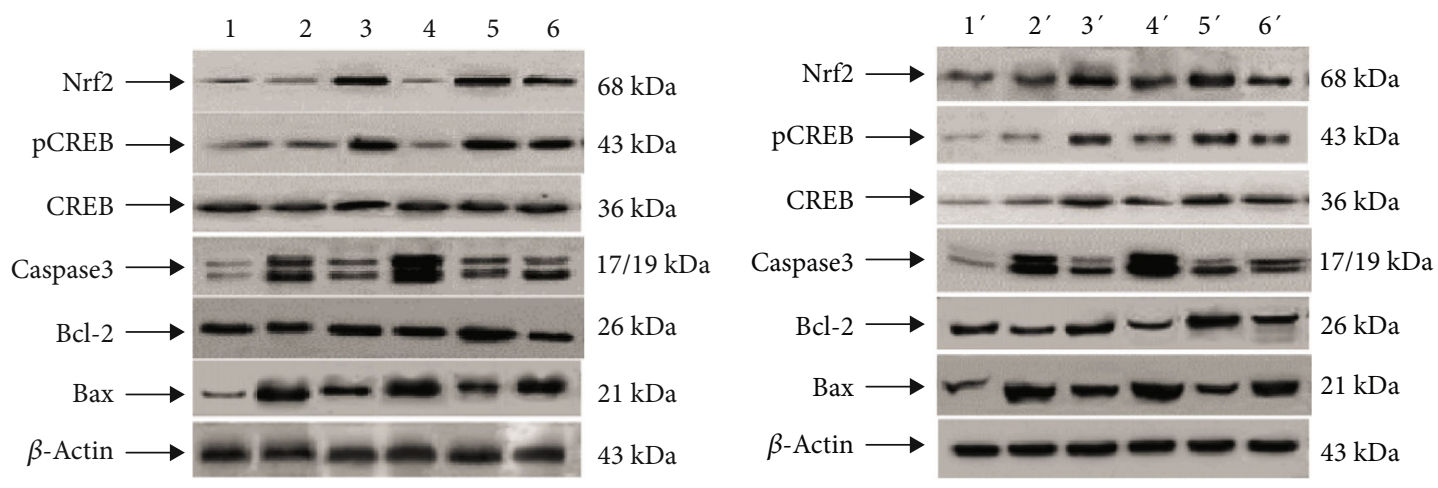

(a)
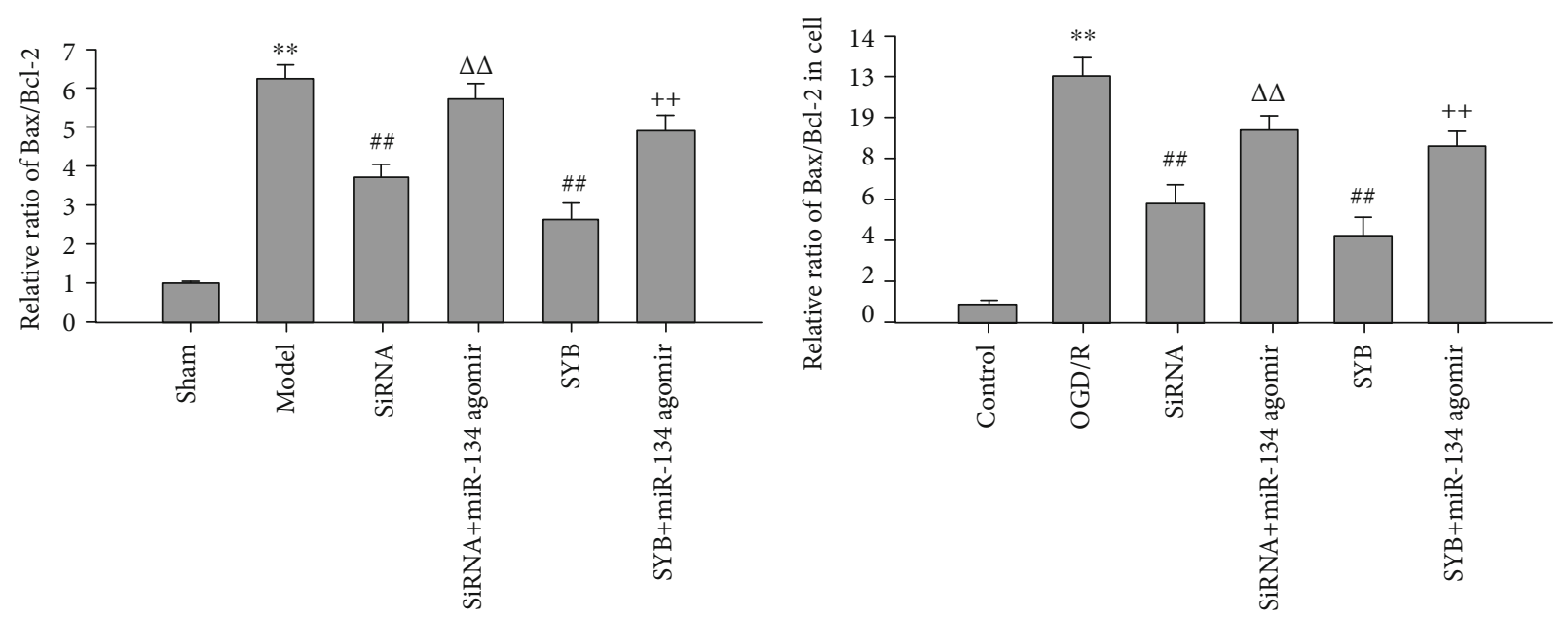

(b)
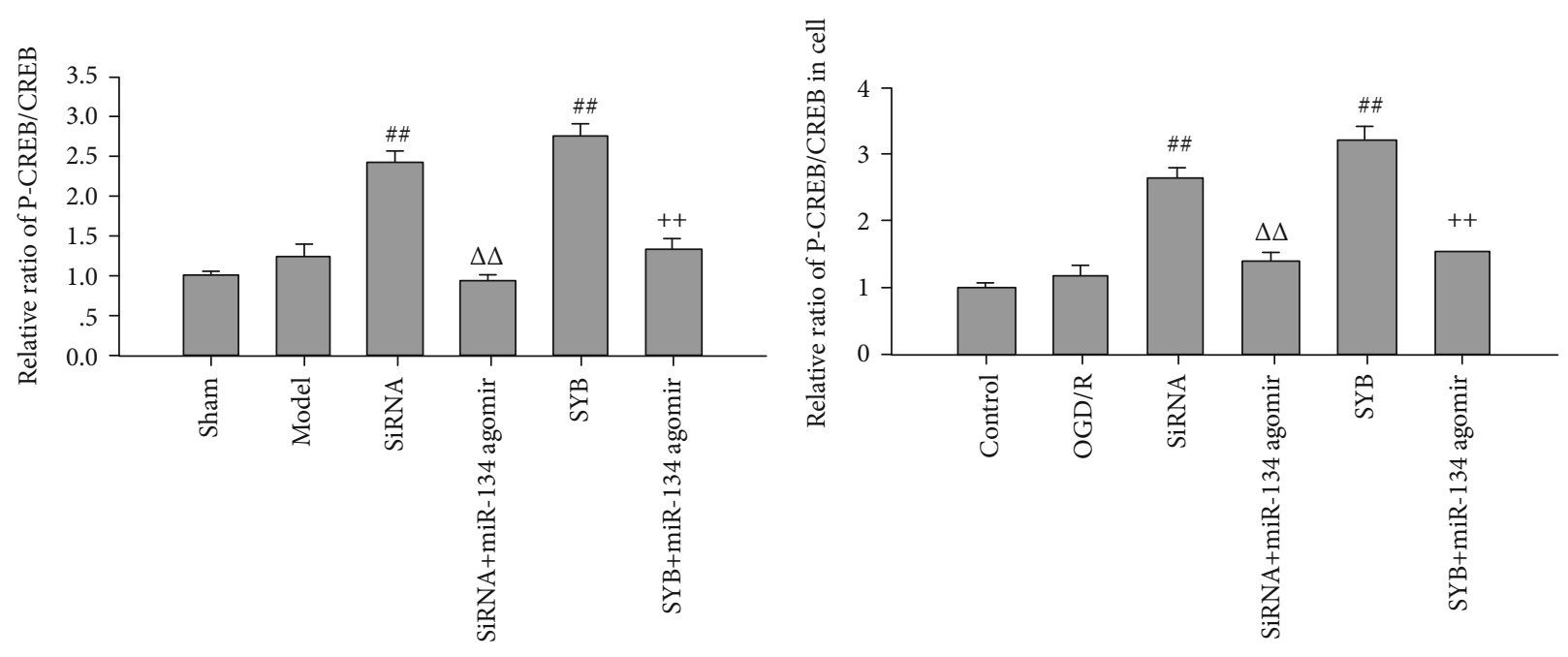

(c)

Figure 6: Continued. 

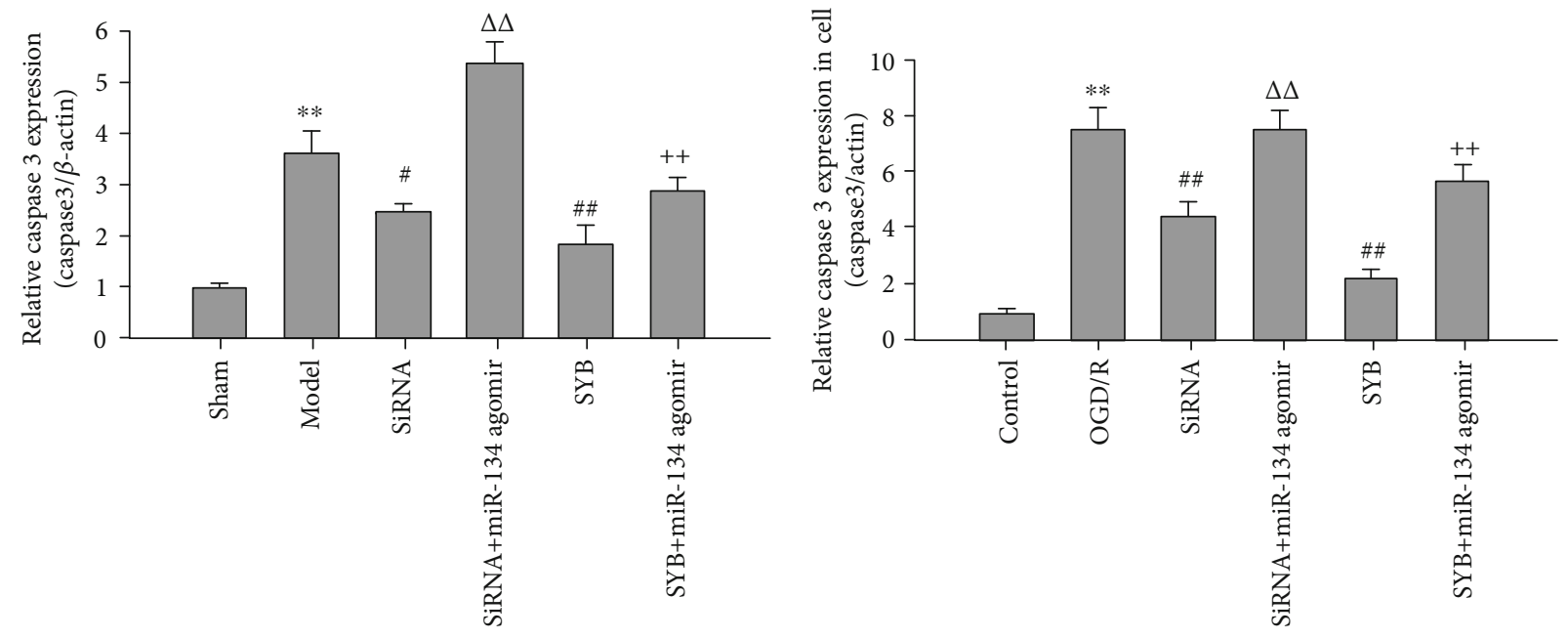

(d)
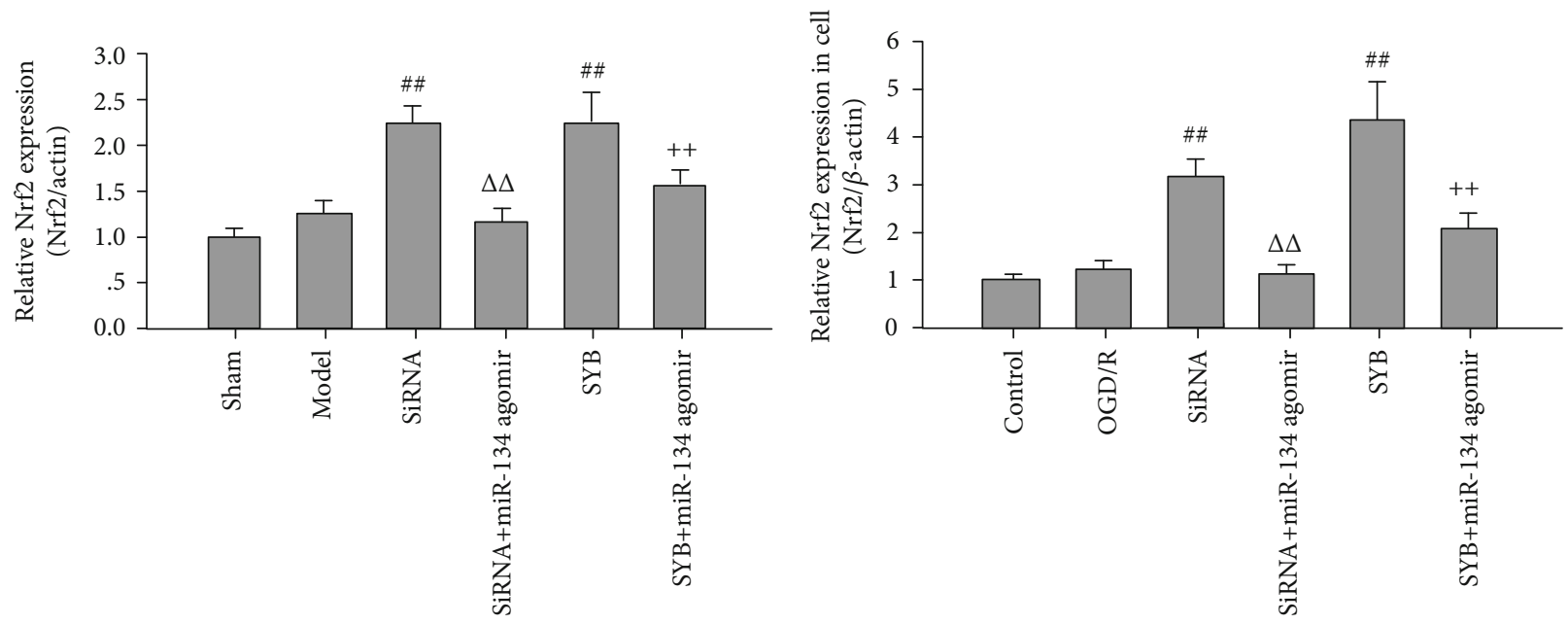

(e)

FIGURE 6: Effect of safflor yellow B on target protein expression. Rats and primary fetal cortical cells were used to establish the I/R model and the OGD/R model, respectively. After I/R for $24 \mathrm{~h}$, total protein in the cerebral cortex and cells from different groups were extracted and measured. Thirty micrograms of protein was loaded per lane and separated by SDS-PAGE and transferred to a PVDF membrane. After incubation with secondary antibodies, the expression levels of CREB, pCREB, Bcl-2, Bax, and Nrf2 were visualized using the chemiluminescence method. (a1) and (a2) represent Western blots (1, 2, 3, 4, 5, and 6 represent sham, ischemia/reperfusion (I/R), AK046177 siRNA, AK046177 siRNA+miR-134 agomir, SYB, and SYB+miR-134 agomir, respectively; $1^{\prime}, 2^{\prime}, 3^{\prime}, 4^{\prime}, 5^{\prime}$, and $6^{\prime}$ represent control, OGD/R, AK046177 siRNA, AK046177 siRNA+miR-134 agomir, SYB, and SYB+miR-134 agomir, respectively). (b1), (c1), (d1), and (e1) represent the relative ratio of Bax/Bcl-2 and pCREB/CREB, caspase3, and Nrf2 expression in the cerebral cortex, respectively. (b2), (c2), (d2), and (e2) represent the relative ratio of Bax/Bcl-2 and pCREB/CREB, caspase3, and Nrf2 expression in cells, respectively. Data are presented as mean $\pm \mathrm{SD}(n=3)$. One-way ANOVA test was used to determine statistical significance. ${ }^{* *} P<0.01 v s$. the sham group or the control group, ${ }^{\#} P<0.05$ or ${ }^{\# \#} P<0.01 v s$. the I/R group or the OGD/R group, ${ }^{\Delta \Delta} P<0.01 v s$. the AK046177 siRNA group, and ${ }^{++} P<0.01 v$ s. the SYB group.

reduces ischemia-mediated brain injury and increases BDNF and Bcl-2 expression [15, 47]. Mabuchi et al. and Huang et al. showed that miR-134 directly targets the $3^{\prime}$-UTR of CREB mRNA, influences the translation and phosphorylation of CREB, and suppresses BDNF and Bcl-2 expression, leading to apoptosis [47]. Conversely, the activation of miR-134 suppresses the expression of CREB and aggravates tissue or cell injury.

Nrf2 is an important transcription factor that can alleviate oxidative stress-mediated cellular damage by activating the transcription of antioxidant enzymes. Nguyen et al. demonstrated the potential of Nrf2-mediated transcription to protect the brain from neurodegeneration resulting from oxidative stress [48]. The phosphorylation of CREB also enhances the binding of Nrf2 to its DNA response element $[49,50]$. In the present study, we found that $\mathrm{I} / \mathrm{R}$ and $\mathrm{OGD} / \mathrm{R}$ induced overexpression of miR-134 and AK046177 and reduced intracellular cAMP levels, which in turn suppressed the expression and phosphorylation of CREB. The result was a further downregulation of Nrf2 and upregulation of Bax 
Control

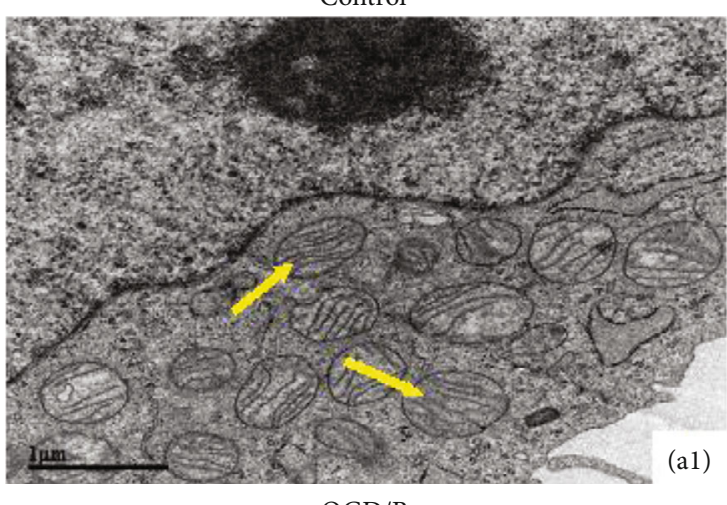

$$
\text { OGD/R }
$$

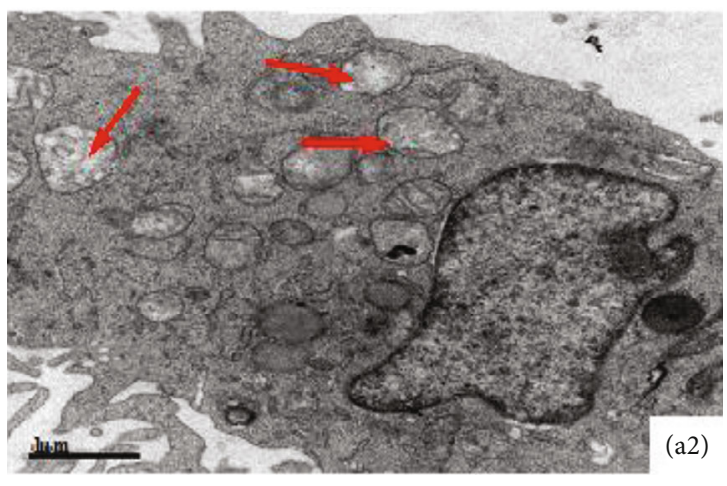

siRNA

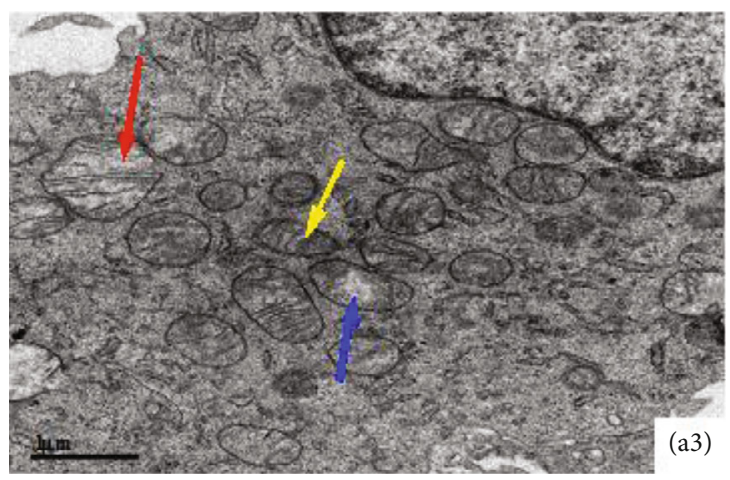

siRNA+miR-134 agomir

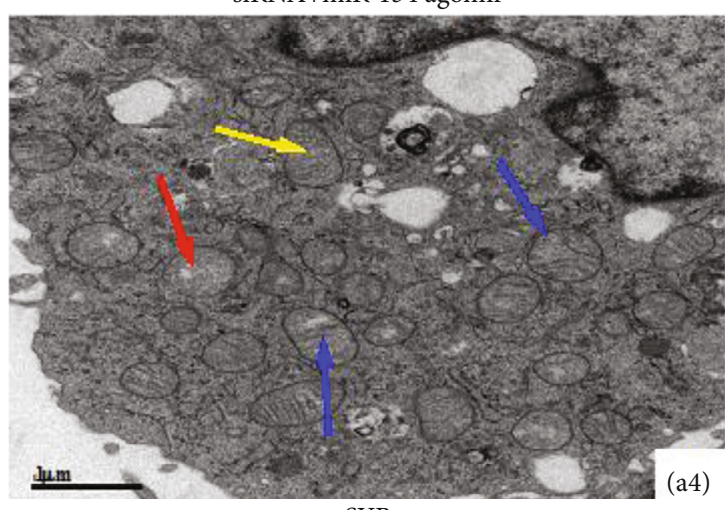

SYB

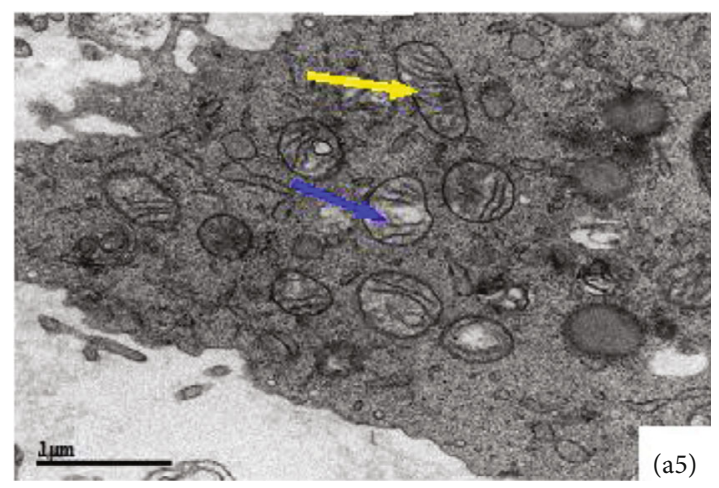

SYB+miR-134 agomir

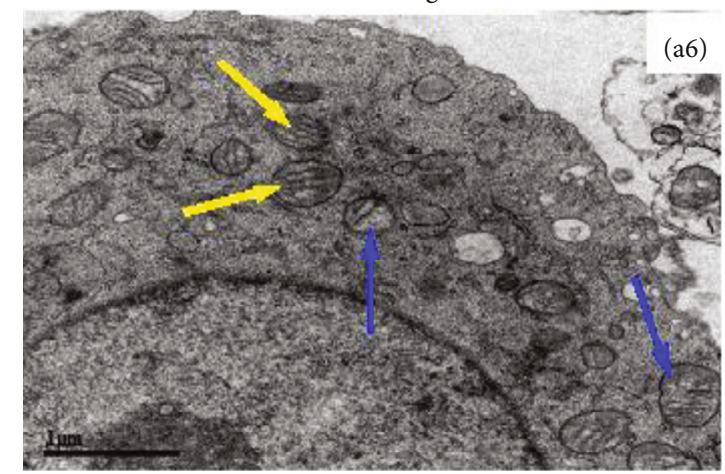

(a6)

(a)

Figure 7: Continued. 


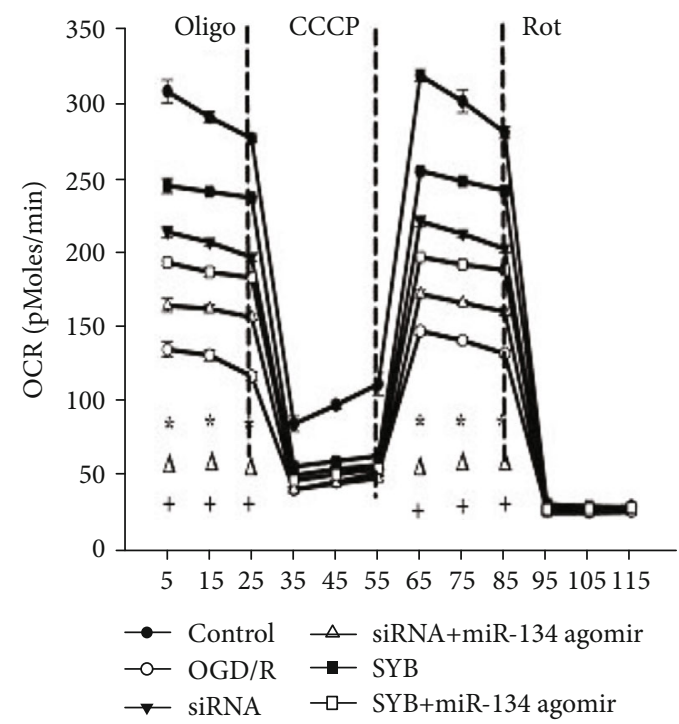

(b)

Figure 7: Effect of safflor yellow B on mitochondrial structure and cell respiration. Primary fetal cortical cells were seeded in 96-well and 6well plates and divided into six groups: control, OGD/R, AK046177 siRNA, AK046177 siRNA+miR-134 agomir, SYB, and SYB+miR-134 agomir. The cells were pretreated with the drugs including SYB, AK046177 siRNA, and miR-134 agomir before being dealing with OGD/R. (a1-a6) Mitochondrial structure was evaluated using a transmission electron microscope $(\times 20,000$ magnification, bar $1 \mu \mathrm{m})$ and represent the mitochondria structure of the control group, the OGD/R group, AK046177 siRNA, AK046177 siRNA+miR-134 agomir, SYB, and SYB+miR-134 agomir, respectively. Transmission electron microscope shows normal mitochondrial structure (yellow solid line arrows), and pathological mitochondria with irregular shapes and swollen (red solid line arrow) and vesicular mitochondrial clusters (blue solid line arrow). Cellular oxygen consumption rate (OCR) was measured using an Oxygraph-2k system ( $n=3$ experiments per condition). Data are presented as mean $\pm \mathrm{SD}(n=3)$. One-way ANOVA test was used to determine statistical significance. ${ }^{*} P<0.01 v s$. the control group, ${ }^{\#} P<0.01 v$ s. the OGD/R group, ${ }^{\Delta} P<0.01 v$ s. the AK046177 siRNA group, ${ }^{+} P<0.01 v s$. the SYB group.

and caspase3, eventually leading to oxidative damage and apoptosis.

Inhibition of AK046177 increased cAMP synthesis and promoted the expression and phosphorylation of CREB, and then stimulated Nrf2 activation and effectively attenuated I/R- or OGD/R-mediated injury by the downregulation of miR-134. Previous studies have shown that the neuroprotective effect of SYB is related to increasing antioxidative enzyme activities, decreasing free radical generation, and regulating Bcl-2/Bax signaling [24], each of which was confirmed in the present study. Moreover, our data demonstrated that SYB also improved cAMP synthesis, and then enhanced the ratio of pCREB/CREB and activated Nrf2 expression via the suppression of overexpressions of AK046177 and miR-134 induced by I/R or OGD/R.

Increased mitochondrial oxidative stress induces mitochondrial dysfunction, which has been linked to a variety of diseases including cardiovascular disease [51, 52]. Mitochondrial dysfunction increases ROS due to electrons escaping from the mitochondrial respiratory chain and interacting with oxygen molecules. ROS destroy mitochondrial membranes and influence cell respiration, facilitate the release of cytochrome $\mathrm{C}$, and promote activation of caspase3, ultimately leading to apoptosis $[53,54]$.

Nrf2 is a prominent player in supporting the structural and functional integrity of the mitochondria under conditions of stress, and thus plays a crucial role in the mainte- nance of cellular redox homeostasis. It regulates the mitochondrial production of ROS and NADPH oxidase [55]. In the present study, we discovered that I/R and OGD/R caused mitochondrial structural damage, such as an increase in mitochondrial volume and the formation of fractured cristae, and increased NADPH oxidase activity. This damage resulted in mitochondrial dysfunction, demonstrated by lower OCR and ROS generation. Both AK046177 siRNA and SYB were able to increase the number of healthy mitochondria, dramatically enhance cellular respiratory function, and inhibit ROS production. However, miR-134 agomir remarkably increased ROS levels by blocking the mitochondrial respiratory chain and reducing the increased activity of antioxidant enzymes related to CREB binding, thereby boosting apoptosis.

\section{Conclusion}

In this study, SYB protected the brain from I/R-related injury by increasing intracellular cAMP levels, downregulating overexpressions of AK046177 and miR-134, and activating the CREB/Nrf2 pathway. The mechanisms regulating I/R injury are complicated, involving a variety of miRNAs and signaling pathways $[35,56]$. We believed that these data only reflected one aspect of the event, and some important proteins may contribute to the understanding of the mechanisms involved in I/R-mediated neuronal injury. 


\author{
Abbreviations \\ SYB: $\quad$ Safflor yellow B \\ ROS: $\quad$ Reactive oxygen species \\ CREB: $\quad$ Cyclic AMP (cAMP) response element-binding \\ protein \\ Nrf2: $\quad$ Nuclear factor erythroid 2-related factor 2 \\ AK046177: Long noncoding RNA046177 \\ miR-134: MicroRNA-134 \\ I/R: Ischemia/reperfusion \\ OGD/R: Oxygen-glucose deprivation \\ CAMP: Cyclic AMP \\ ARE: Antioxidant response elements \\ BDNF: Brain-derived neurotrophic factor \\ DMEM: Dulbecco's minimal essential medium \\ MCAO: Middle cerebral artery occlusion \\ SOD: $\quad$ Superoxide dismutase \\ GPx: $\quad$ Glutathione peroxidase \\ MDA: Malondialdehyde \\ HSYA: Hydroxysafflor yellow A \\ OCR: Oxygen consumption rate \\ CCCP: Carbonyl cyanide chlorophenylhydrazine \\ OXPHOS: Oxidative phosphorylation \\ MTT: 3-(4,5-dimethyithiazol-2-yl)-2,5-diphenyl-tet- \\ razolium bromide.
}

\section{Data Availability}

The data is available on request by asking directly the corresponding author by mail at ytwcy@163.com.

\section{Conflicts of Interest}

The authors declared no potential conflicts of interest with respect to the research, authorship, and/or publication of this article.

\section{Authors' Contributions}

C.W. conceived and designed the study. H.W., Y.S., K.W., and H.S. performed the apoptosis and cell viability assays, Western blot analysis, and qRT-PCR. H.W., Q.W., and Y.S. observed mitochondrial morphology and cell respiration. H.W., H.S., and Q.W. detected the levels of ROS, MDA, cAMP, and enzyme activity. Q.W., Y.S., C.Z., and H.S. performed the statistical analyses. C.W. and C.Z. wrote the manuscript. Hongzhi Wan contributed equally to this work.

\section{Acknowledgments}

This study was supported by the Natural Science Foundation of Shandong Province (ZR2012HM076), the National Natural Science Foundation of China (81530030, 81670398, and 91639102), the Foundation for Outstanding Young Scientist in Shandong Province (BS2014YY049), and the Science and Technology Project of Yantai (2014ZH092).

\section{References}

[1] D. Lloyd-Jones, R. J. Adams, T. M. Brown et al., "Heart disease and stroke statistics-2010 update," Circulation, vol. 121, no. 7, pp. e46-e215, 2010.

[2] T. A. Burke and R. N. Venketasubramanian, "The epidemiology of stroke in the East Asian region: a literature based review," International Journal of Stroke, vol. 1, no. 4, pp. 208-215, 2016.

[3] T. Truelsen, S. Begg, and C. Mathers, "The global burden of cerebrovascular disease," 2006, https://www.researchgate.net/ publication/228551377.

[4] E. H. Lo, T. Dalkara, and M. A. Moskowitz, "Mechanisms, challenges and opportunities in stroke," Nature Reviews. Neuroscience, vol. 4, no. 5, pp. 399-414, 2003.

[5] M. Y.-. T. Globus, R. Busto, B. Lin, H. Schnippering, and M. D. Ginsberg, "Detection of free radical activity during transient global ischemia and recirculation: effects of intraischemic brain temperature modulation," Journal of Neurochemistry, vol. 65 , no. 3, pp. 1250-1256, 1995.

[6] C. A. Piantadosi and J. Zhang, "Mitochondrial generation of reactive oxygen species after brain ischemia in the rat," Stroke, vol. 27, no. 2, pp. 327-332, 1996.

[7] L. Ste-Marie, P. Vachon, L. Vachon, C. Bémeur, M. C. Guertin, and J. Montgomery, "Hydroxyl radical production in the cortex and striatum in a rat model of focal cerebral ischemia," The Canadian Journal of Neurological Sciences, vol. 27, no. 2, pp. 152-159, 2000.

[8] T. Yamamoto, S. Yuki, T. Watanabe, M. Mitsuka, K. I. Saito, and K. Kogure, "Delayed neuronal death prevented by inhibition of increased hydroxyl radical formation in a transient cerebral ischemia," Brain Research, vol. 762, no. 1-2, pp. 240-242, 1997.

[9] Z. F. Yu, A. J. Bruce-Keller, Y. Goodman, and M. P. Mattson, "Uric acid protects neurons against excitotoxic and metabolic insults in cell culture, and against focal ischemic brain injury in vivo," Journal of Neuroscience Research, vol. 53, no. 5, pp. 613-625, 1998.

[10] H. Imai, H. Masayasu, D. Dewar, D. I. Graham, and I. M. Macrae, "Ebselen protects both gray and white matter in a rodent model of focal cerebral ischemia," Stroke, vol. 32, no. 9, pp. 2149-2154, 2001.

[11] N. Matsumiya, R. C. Koehler, J. R. Kirsch, and R. J. Traystman, "Conjugated superoxide dismutase reduces extent of caudate injury after transient focal ischemia in cats," Stroke, vol. 22, no. 9, pp. 1193-1200, 1991.

[12] J. H. No, Y. B. Kim, and Y. S. Song, "Targeting Nrf2 signaling to combat chemoresistance," Journal of Cancer Prevention, vol. 19, no. 2, pp. 111-117, 2014.

[13] D. D. Zhang and M. Hannink, "Distinct cysteine residues in Keap1 are required for Keap1 dependent ubiquitination of Nrf2 and for stabilization of Nrf2 by chemopreventive agents and oxidative stress," Molecular and Cellular Biology, vol. 23, no. 22, pp. 8137-8151, 2003.

[14] A. Riccio, S. Ahn, C. M. Davenport, J. A. Blendy, and D. D. Ginty, "Mediation by a CREB family transcription factor of NGF-dependent survival of sympathetic neurons," Science, vol. 286, no. 5448, pp. 2358-2361, 1999.

[15] K. Kitagawa, "CREB and cAMP response element-mediated gene expression in the ischemic brain," The FEBS Journal, vol. 274, no. 13, pp. 3210-3217, 2007. 
[16] Y. B. Ouyang, C. M. Stary, R. E. White, and R. G. Giffard, “The use of microRNAs to modulate redox and immune response to stroke," Antioxidants \& Redox Signaling, vol. 22, no. 2, pp. 187-202, 2015.

[17] W. Huang, X. Liu, J. Cao et al., "miR-134 regulates ischemia/ reperfusion injury-induced neuronal cell death by regulating CREB signaling," Journal of Molecular Neuroscience, vol. 55, no. 4, pp. 821-829, 2015.

[18] C. Gong and L. E. Maquat, "IncRNAs transactivate STAU1mediated mRNA decay by duplexing with $3^{\prime}$ UTRs via Alu elements," Nature, vol. 470, no. 7333, pp. 284-288, 2011.

[19] C. Kanduri, "Kcnq1ot1: a chromatin regulatory RNA," Seminars in Cell \& Developmental Biology, vol. 22, no. 4, pp. 343-350, 2011.

[20] D. Khaitan, M. E. Dinger, J. Mazar et al., "The melanomaupregulated long noncoding RNA Spry4-It1 modulates apoptosis and invasion," Cancer Research, vol. 71, no. 11, pp. 3852-3862, 2011.

[21] S. Ramagiri and R. Taliyan, "Neuroprotective effect of hydroxy safflor yellow A against cerebral ischemia-reperfusion injury in rats: putative role of mPTP," Journal of Basic and Clinical Physiology and Pharmacology, vol. 27, no. 1, pp. 1-8, 2016.

[22] J. Cao, Z. Chen, Y. Zhu et al., "Huangqi-Honghua combination and its main components ameliorate cerebral infarction with Qi deficiency and blood stasis syndrome by antioxidant action in rats," Journal of Ethnopharmacology, vol. 155, no. 2, pp. 1053-1060, 2014.

[23] C. Wang, Q. Huang, C. Wang et al., "Hydroxysafflor yellow A suppress oleic acid-induced acute lung injury via protein kinase A," Toxicology and Applied Pharmacology, vol. 272, no. 3, pp. 895-904, 2013.

[24] C. Wang, D. Zhang, G. Li et al., "Neuroprotective effects of safflor yellow B on brain ischemic injury," Experimental Brain Research, vol. 177, no. 4, pp. 533-539, 2007.

[25] C. Wang, H. Ma, S. Zhang, Y. Wang, J. Liu, and X. Xiao, "Safflor yellow B suppresses pheochromocytoma cell (PC12) injury induced by oxidative stress via antioxidant system and Bcl-2/Bax pathway," Naunyn-Schmiedeberg's Archives of Pharmacology, vol. 380, no. 2, pp. 135-142, 2009.

[26] E. Z. Longa, P. R. Weinstein, S. Carlson, and R. Cummins, "Reversible middle cerebral artery occlusion without craniectomy in rats," Stroke, vol. 20, no. 1, pp. 84-91, 1989.

[27] I. M. Macrae, "New models of focal cerebral ischemia," British Journal of Clinical Pharmacology, vol. 34, pp. 302-308, 1992.

[28] D. J. Combs and L. G. D'Alecy, "Motor performance in rats exposed to severe forebrain ischemia: effect of fasting and 1,3-butanediol," Stroke, vol. 18, no. 2, pp. 503-511, 1987.

[29] H. Zhao, R. Wang, Z. Tao et al., "Ischemic postconditioning relieves cerebral ischemia and reperfusion injury through activating T-LAK cell-originated protein kinase/protein kinase B pathway in rats," Stroke, vol. 45, no. 8, pp. 24172424, 2014.

[30] Y. B. Ouyang, Y. Lu, S. Yue et al., "miR-181 regulates GRP78 and influences outcome from cerebral ischemia in vitro and in vivo," Neurobiology of Disease, vol. 45, no. 1, pp. 555-563, 2012.

[31] K. J. Livak and T. D. Schmittgen, "Analysis of relative gene expression data using real-time quantitative PCR and the $2^{-\Delta \Delta \mathrm{CT}}$ method," Methods, vol. 25, no. 4, pp. 402-408, 2001.

[32] D. Pesta and E. Gnaiger, "High-resolution respirometry: OXPHOS protocols for human cells and permeabilized fibers from small biopsies of human muscle," Methods in Molecular Biology, vol. 810, pp. 25-58, 2012.

[33] D. P. Bartel, "MicroRNAs: genomics, biogenesis, mechanism, and function," Cell, vol. 116, no. 2, pp. 281-297, 2004.

[34] J. T. Mendell and E. N. Olson, "MicroRNAs in stress signaling and human disease," Cell, vol. 148, no. 6, pp. 1172-1187, 2012.

[35] J. Winter, S. Jung, S. Keller, R. I. Gregory, and S. Diederichs, "Many roads to maturity: microRNA biogenesis pathways and their regulation," Nature Cell Biology, vol. 11, no. 3, pp. 228-234, 2009.

[36] Y. Di, Y. Lei, F. Yu, F. Changfeng, W. Song, and M. Xuming, "MicroRNAs expression and function in cerebral ischemia reperfusion injury," Journal of Molecular Neuroscience, vol. 53, no. 2, pp. 242-250, 2014.

[37] C. Liu, Z. Peng, N. Zhang et al., "Identification of differentially expressed microRNAs and their PKC-isoform specific gene network prediction during hypoxic pre-conditioning and focal cerebral ischemia of mice," Journal of Neurochemistry, vol. 120, no. 5, pp. 830-841, 2012.

[38] S. Wang, W. Yu, J. Chen, T. Yao, and F. Deng, "IncRNA MALAT1 sponges miR-203 to promote inflammation in myocardial ischemia-reperfusion injury," International Journal of Cardiology, vol. 268, p. 245, 2018.

[39] S. B. Ong, K. Katwadi, X. Y. Kwek et al., "Non-coding RNAs as therapeutic targets for preventing myocardial ischemiareperfusion injury," Expert Opinion on Therapeutic Targets, vol. 22, no. 3, pp. 247-261, 2018.

[40] H. Yan, J. Rao, J. Yuan et al., "Long non-coding RNA MEG3 functions as a competing endogenous RNA to regulate ischemic neuronal death by targeting miR-21/PDCD4 signaling pathway," Cell Death \& Disease, vol. 8, no. 12, p. 3211, 2017.

[41] E. Fuentes, L. Badimon, J. Caballero et al., "Protective mechanisms of adenosine $5^{\prime}$-monophosphate in platelet activation and thrombus formation," Thrombosis and Haemostasis, vol. 111, no. 3, pp. 491-507, 2017.

[42] M. Ghosh, Y. Xu, and D. D. Pearse, "Cyclic AMP is a key regulator of M1 to M2a phenotypic conversion of microglia in the presence of Th2 cytokines," Journal of Neuroinflammation, vol. 13, no. 1, p. 19, 2016.

[43] S. Saxena, R. E. Rönn, C. Guibentif, R. Moraghebi, and N. B. Woods, "Cyclic AMP signaling through Epac axis modulates human hemogenic endothelium and enhances hematopoietic cell generation," Stem Cell Reports, vol. 6, no. 5, pp. 692-703, 2016.

[44] N. Li, B. Li, T. Brun et al., "NADPH oxidase NOX2 defines a new antagonistic role for reactive oxygen species and cAMP/PKA in the regulation of insulin secretion," Diabetes, vol. 61, no. 11, pp. 2842-2850, 2012.

[45] B. R. Hu, C. M. Fux, M. E. Martone, J. A. Zivin, and M. H. Ellisman, "Persistent phosphorylation of cyclic AMP responsive element-binding protein and activating transcription factor-2 transcription factors following transient cerebral ischemia in rat brain," Neuroscience, vol. 89, no. 2, pp. 437452, 1999.

[46] K. Tanaka, "Alteration of second messengers during acute cerebral ischemia-adenylate cyclase, cyclic AMP-dependent protein kinase, and cyclic AMP response element binding protein," Progress in Neurobiology, vol. 65, no. 2, pp. 173207, 2001.

[47] T. Mabuchi, K. Kitagawa, K. Kuwabara et al., "Phosphorylation of cAMP response element-binding protein in 
hippocampal neurons as a protective response after exposure to glutamate in vitro and ischemia in vivo," The Journal of Neuroscience, vol. 21, no. 23, pp. 9204-9213, 2001.

[48] T. Nguyen, P. Nioi, and C. B. Pickett, "The Nrf2-antioxidant response element signaling pathway and its activation by oxidative stress," The Journal of Biological Chemistry, vol. 284, no. 20, pp. 13291-13295, 2009.

[49] F. Moosavi, R. Hosseini, L. Saso, and O. Firuzi, "Modulation of neurotrophic signaling pathways by polyphenols," Drug Design, Development and Therapy, vol. 10, pp. 23-42, 2015.

[50] M. C. Chiang, H. Lin, Y. C. Cheng, C. H. Yen, R. N. Huang, and K. H. Lin, "Beta-adrenoceptor pathway enhances mitochondrial function in human neural stem cells via rotary cell culture system," Journal of Neuroscience Methods, vol. 207, no. 2, pp. 130-136, 2012.

[51] H. J. Ku, Y. Ahn, J. H. Lee, K. M. Park, and J. W. Park, "IDH2 deficiency promotes mitochondrial dysfunction and cardiac hypertrophy in mice," Free Radical Biology \& Medicine, vol. 80, pp. 84-92, 2015.

[52] M. T. Lin and M. F. Beal, "Mitochondrial dysfunction and oxidative stress in neurodegenerative diseases," Nature, vol. 443, no. 7113, pp. 787-795, 2006.

[53] J. Z. Li, S. Y. Yu, D. Mo, X. N. Tang, and Q. R. Shao, "Picroside II inhibits hypoxia/reoxygenation-induced cardiomyocyte apoptosis by ameliorating mitochondrial function through a mechanism involving a decrease in reactive oxygen species production," International Journal of Molecular Medicine, vol. 35, no. 2, pp. 446-452, 2015.

[54] K. M. Hung and M. J. Calkins, "Mitochondrial homeostatic disruptions are sensitive indicators of stress in neurons with defective mitochondrial DNA transactions," Mitochondrion, vol. 31, pp. 9-19, 2016.

[55] A. T. Dinkova-Kostova and A. Y. Abramov, "The emerging role of Nrf2 in mitochondrial function," Free Radical Biology \& Medicine, vol. 88, no. Part B, pp. 179-188, 2015.

[56] V. P. Nakka, A. Gusain, S. L. Mehta, and R. Raghubir, "Molecular mechanisms of apoptosis in cerebral ischemia: multiple neuroprotective opportunities," Molecular Neurobiology, vol. 37, no. 1, pp. 7-38, 2008. 